\title{
Anisotropic magnetism in field-structured composites
}

\author{
James E. Martin, Eugene Venturini, Judy Odinek and Robert A. Anderson \\ Sandia National Laboratories
}

Albuquerque, New Mexico 87185-1421

\section{RECEIVED \\ JUL 131999 \\ OSTI}

Abstract: Magnetic field-structured-composites (FSCs) are made by structuring magnetic particle suspensions in uniaxial or biaxial (e.g. rotating) magnetic fields, while polymerizing the suspending resin. A uniaxial field produces chain-like particle structures, and a biaxial field produces sheet-like particle structures. In either case, these anisotropic structures affect the measured magnetic hysteresis loops, with the magnetic remanence and susceptibility increased significantly along the axis of the structuring field, and decreased slightly orthogonal to the structuring field, relative to the unstructured particle composite. The coercivity is essentially unaffected by structuring. We present data for FSCs of magnetically soft particles, and demonstrate that the altered magnetism can be accounted for by considering the large local fields that occur in FSCs. FSCs of magnetically hard particles show unexpectedly large anisotropies in the remanence, and this is due to the local field effects in combination with the large crystalline anisotropy of this material.

Sandia is a multiprogram laboratory operated by Sandia Corporation, a Lockheed Martin Company, for the United States Department of Energy under contract DE-AC04-94AL8500. This work supported by the Division of Materials Sciences, Office of Basic Energy Sciences, U.S. Department of Energy (DOE). 


\section{DISCLAIMER}

This report was prepared as an account of work sponsored by an agency of the United States Government. Neither the United States Government nor any agency thereof, nor any of their employees, make any warranty, express or implied, or assumes any legal liability or responsibility for the accuracy, completeness, or usefulness of any information, apparatus, product, or process disclosed, or represents that its use would not infringe privately owned rights. Reference herein to any specific commercial product, process, or service by trade name, trademark, manufacturer, or otherwise does not necessarily constitute or imply its endorsement, recommendation, or favoring by the United States Government or any agency thereof. The views and opinions of authors expressed herein do not necessarily state or reflect those of the United States Government or any agency thereof. 


\section{DISCLAIMER}

Portions of this document may be illegible in electronic image products. Images are produced from the best available original document. 


\section{INTRODUCTION}

When a magnetic particle suspension, consisting of multi-domain particles, is exposed to a uniaxial magnetic field, the magnetic dipole moment on the particles will generally increase and align with the applied field. The particles will then migrate under the influence of the dipolar interactions with neighboring particles, to form complex chainlike structures [1]. If a magnetic particle suspension is instead exposed to a biaxial (e.g. rotating) magnetic field, the induced dipole moments create a net attractive interaction in the plane of the field, resulting in the formation of complex sheet-like structures [2]. Similar effects occur when suspensions of dielectric particles are subjected to uniaxial and biaxial electric fields. These materials, which we call field-structured composites (FSCs), can have large anisotropies in properties such as their conductivity, permittivity, dielectric breakdown strength, optical transmittance, etc.

In this paper we report on the magnetic hysteresis loops of uniaxial and biaxial FSCs of magnetic particles in a thermosetting resin. Relative to unstructured particle composites, the susceptibility and remanence of these materials is increased along the axis of the structuring field, and decreased orthogonal to this, yet the coercivity is not strongly affected by structuring. We demonstrate that in some cases these changes in the magnetization curves can be attributed to structurally-induced changes in the local magnetic field, which we compute for simulated FSC structures, obtained from large scale dynamical simulations of interacting hard sphere dipoles. Other contributing factors include the crystalline magnetic anisotropy and the shape anisotropy of the particles. By considering invariants that involve the susceptibility, we can distinguish between these factors experimentally.

\section{EXPERIMENTAL}

\section{Sample preparation}

Both magnetically soft and hard particles were used in these studies. The soft magnetic particles were a 3-5 $\mu \mathrm{m}$ carbonyl iron powder from ISP Technologies ${ }^{\mathrm{TM}}$ (ISP-R2430), 
and was used to make samples with concentrations in the range of $2.0-30.0$ vol. \%. The magnetically hard particles were made of $\mathrm{SmCo}_{5}$, and were used at a concentration of 2.5 vol. \%. Finally, stainless steel fibers from Bekaert Fibre Technologies ${ }^{\mathrm{TM}}$ were used at a concentration of $1.55 \mathrm{vol} . \%$. These fibers are $1.0 \mu \mathrm{m}$ diameter and $1 \mathrm{~mm}$ in length.

The particles were suspended in a mixture of the epoxy resin Epon $828^{\mathrm{TM}}$ using $1.0 \mathrm{wt} \%$ Triton $\mathrm{X}-100^{\mathrm{TM}}$ as a dispersant. These suspensions were placed in an ultrasonic bath for $1 \mathrm{hr}$, and the amine hardener, a 50/50 mixture of Ancamine/Jeffamine, ${ }^{\mathrm{TM}}$ was stirred into the suspension. The suspensions were then placed in $1 \mathrm{~cm}$ square polystyrene cuvets and degassed in a vacuum oven at $50^{\circ} \mathrm{C}$ for 10 minutes. A $150 \mathrm{G}$ magnetic field was supplied by two large plate magnets oriented such that the magnetic field was vertical, to prevent the sedimentation of evolving chain structures. To create a uniform biaxial field, we simply mounted the square cuvet on a motor and rotated it around the four-fold axis at a speed of $100 \mathrm{rpm}$ and plunged the sample into the center of the magnets. Of course, one can create a rotating field with orthogonal coils in quadrature phase, but rotating the sample actually works quite well.

Curing the samples was done in several stages. The random particle dispersions, which are our control samples, were cured at $25^{\circ} \mathrm{C}$ for $20 \mathrm{hrs}$ while being rotated around the horizontal four-fold axis, to prevent sedimentation. This gels the resin so that the particles cannot sediment, but does not fully cure the epoxy. To fully cure the epoxy, we then post-cured by ramping from $50^{\circ} \mathrm{C}$ to $100^{\circ} \mathrm{C}$ over $3 \mathrm{hrs}$, and then curing at $100^{\circ} \mathrm{C}$ for at least 3 more hrs. The biaxial samples are made the same way, but the initial, $25^{\circ} \mathrm{C}$ cure was done in a rotating magnetic field. The uniaxial samples were placed in a uniaxial magnetic field and cured for $20 \mathrm{hrs}$ at $50^{\circ} \mathrm{C}$ to gel the resin. A post-cure was done by ramping from $50^{\circ} \mathrm{C}$ to $100^{\circ} \mathrm{C}$ over $3 \mathrm{hrs}$, and curing at $100^{\circ} \mathrm{C}$ for at least 3 more hrs. These samples were then sectioned with a diamond saw for susceptibility measurements parallel and orthogonal to the direction of the structuring field. Optical images of these structures are shown in Fig. 1. 


\section{Magnetic measurements}

Isothermal magnetic hysteresis data were measured at room temperature $(293 \mathrm{~K}$ ) for applied fields between +1 and $-1 \mathrm{~T}\left(+6\right.$ and $-6 \mathrm{~T}$ for $\left.\mathrm{SmCo}_{5}\right)$ using a commercial SQUID magnetometer with extended dynamic range. The extended range allowed the use of relatively large random and FSC rectangular samples (typically $3 \times 3 \times 5 \mathrm{~mm}^{3}$ ) with saturation moments up to $25 \mathrm{~mA} \mathrm{~m}^{2}$ (25 emu) for $30 \mathrm{vol} \%$ iron. These dimensions are much larger than the coarseness of the field structuring shown in Fig. 1, assuring a representative result. At the maximum field of $1 \mathrm{~T}$ these samples were in the reversible approach-to-saturation regime, minimizing any history effects in the measurements.

\section{Simulations}

We have reported athermal [1,2] and thermal [3] simulation studies of structure formation in field-structured composites. In this paper we use the results of those simulations as model structures for the computation of the local field effects that we believe are responsible for the susceptibility data.

Briefly, in these Langevin dynamics simulations the particles are essentially hard spheres with induced dipolar interactions, Stokes friction against the solvent, and Brownian motion. The structures used in this paper are obtained from a simulation method developed to predict the evolution of large, $N=10,000$ particle systems over short times (in general, $\leq 150$ dimensionless time units). This method has time complexity $O(N)$, but gives structures that are statistically indistinguishable from a separate, more direct $O\left(N^{2}\right)$ simulation developed to predict the evolution of smaller systems over longer times. In most of the simulations cyclic boundary conditions are used in all directions. The size of the simulations led to structures whose scale of coarseness was much smaller than the simulation volume, minimizing the effect of the cyclic boundary conditions. Representative simulation results for athermal simulations in uniaxial and biaxial fields are shown in Fig. 2. 
Dipolar force. It is helpful to discuss the dipolar forces used in our uniaxial and biaxial simulations in detail. We adopt the convention that the uniaxial field is applied along the $z$ axis, $\mathbf{H}_{0}=H_{0} \hat{\mathbf{z}}$, and the biaxial field is a rotating field in the $x-y$ plane, $\mathbf{H}_{0}=H_{0}[\sin (\omega t) \hat{\mathbf{x}}+\cos (\omega t) \hat{\mathbf{y}}]$, where the field frequency is assumed sufficiently high that we need only consider the force of interaction averaged over a field cycle. In either case, the dipole-dipole interaction force between two spheres whose center of mass separation vector $\mathbf{r}$ is of length $r$ and is inclined at an angle $\theta$ to the $z$ axis is

$$
\mathbf{F}_{d}(r, \theta)=-\alpha C\left(\frac{d}{r}\right)^{4}\left[\left(3 \cos ^{2} \theta-1\right) \hat{r}+\sin 2 \theta \hat{\theta}\right]
$$

where $\alpha=1$ for the uniaxial field and $\alpha=-1 / 2$ for the biaxial field. For spherical particles of radius $a$, the constant $C=\frac{3}{16} \frac{\mu_{o} \kappa_{c}|\mathbf{m}|^{2}}{4 \pi a^{4}}$, where $\mathbf{m}$ is the magnetic dipole moment, which we take to be aligned with the instantaneous field, as justified forthwith [4]. Here $\kappa_{c}$ is the relative permeability (to the vacuum permeability $\mu_{o}=4 \pi \times 10^{-7} \mathrm{H} / \mathrm{m}$ ) of the continuous polymeric phase, which in practice is very nearly 1 . In the absence of Brownian motion, the constant $C$ determines only the timescale of structural evolution, and is specifically not a parameter that can be used to control structure.

Brownian motion. To compare simulation to experiment requires a reasonable estimate of the magnitude of thermal forces relative to the dipolar forces. For paramagnetic or diamagnetic spherical particles the induced magnetic moment is $\mathbf{m}=4 \pi a^{3} \beta \mathbf{H}_{0}$ where $\beta=\left(\kappa_{p}-\kappa_{c}\right) /\left(\kappa_{p}+2 \kappa_{c}\right)$ is the permeability contrast factor, and $\kappa_{p}$ is the relative permeability of the particles [4]. The force constant in this case is $C=\frac{3 \pi}{4} \mu_{o} \kappa_{c} a^{2} \beta^{2}\left|\mathbf{H}_{a}\right|^{2}$.

For ferromagnetic particles the magnetic dipole moment depends on the field history as well as the applied field. If the particles are initially unpoled, then at low applied fields they will act as paramagnetic particles having a high permeability. On the other hand, if such particles are exposed to a saturating magnetic field $\mathbf{H}_{\mathrm{s}}$ the domain 
walls will move to create a dipole moment that is roughly $\mathbf{m}_{s}=\frac{4 \pi}{3} a^{3} \mathbf{M}_{s}$, where $\mathbf{M}_{\mathbf{s}}$ is the saturation magnetization along the easy axis of the magnetic material. After this poling field is turned off, the dipole moment will decay to $\mathbf{m}_{r}=\frac{4 \pi}{3} a^{3} \mathbf{M}_{r}$, where $\mathbf{M}_{r}$ is the remanent magnetization. This dipole moment can be an order of magnitude larger than that of a high permeability soft magnetic material in the low fields we apply to structure the particles, so we will consider only the latter case in order to find a lower bound to the dipolar interactions.

The minor (low field) hysteresis loops of ferromagnetic materials can be closely approximated as a paramagnetic response with a relative permeability on the order of $\kappa_{p} \approx 10^{4}$. Because the relative permeability of the liquid phase is essentially 1 , this gives $\beta \approx 1$. For a typical magnetic field produced by our structuring magnets, of $H_{0} \cong 12 \times 10^{3}$ $\mathrm{Am}^{-1}(150 \mathrm{Oe})$, and a particle radius of $1 \mu \mathrm{m}$, this gives a dipole moment $m \cong 1.5 \times 10^{-13}$ $\mathrm{Am}^{2}$. The interaction energy of the dipole moment with the applied field will be $\mu_{0} m H_{0}$ $=2.3 \times 10^{-15} \mathrm{~J}$, and because the thermal energy at room temperature is $k_{B} T=4.1 \times 10^{-21}$, the poled particles will be completely aligned with the field, justifying the use of Eq. 1 .

The energy required to separate two magnetized particles in contact along the $z$ axis is $\frac{m^{2} \mu_{0}}{16 \pi a^{3}}=5.6 \times 10^{-16} \mathrm{~J}$, which is vastly in excess of the thermal energy, so that such particles will contact irreversibly and Brownian motion should have a negligible effect on the evolution of structure. Thus the structures we use to compare simulation to experiment are those that have been generated athermally.

Timescale. In the absence of Brownian motion the strength of the dipolar interactions alters only the coarsening timescale, not the structural evolution. The dimensionless numerical equation of motion is thus of the form $\Delta u=\Delta s f(r, \theta)$, where the dimensionless length $\Delta u=\Delta r / 2 a$. The dimensionless time is $\Delta s=\Delta t \mu_{0} \kappa_{c} \beta^{2} H_{o}{ }^{2} / 16 \eta_{o}$. For a suspending liquid with a viscosity $\eta_{0}$ of $1 \mathrm{cp}$, an applied field of $H_{o}=3.58 \mathrm{~A} / \mathrm{m}(45 \mathrm{Oe})$, $\kappa_{c}=1$ and $\beta=1, \Delta s \cong \Delta t(\mathrm{~s}) \times 10^{3}\left(\mathrm{~s}^{-1}\right)$ so under these conditions one dimensionless time unit is about a millisecond. The simulation data we generate are for structures that have 
evolved for 150 dimensionless time units, which is far less than the time over which the experimental samples were allowed to structure.

\section{THEORY}

There are at least three possible causes for the changes in the magnetic properties of field structured composites. The first is that in the magnetic field the particles rotate so that the easy magnetic axis of the particles aligns with the field. The second is that the particles are non-spherical and thus rotate so that their long axis aligns with the field, since this increases their polarizability and thus reduces their free energy. The third is that the local magnetic field is strongly affected by structuring the samples. Depending on the type of particles used, some or all of these effects can be important.

Particles with a polycrystalline morphology, such as carbonyl iron, should not exhibit a pronounced easy axis, due to the averaging over randomly oriented crystalline domains within particles. Furthermore, these particles do not have sufficient shape anisotropy to account for the effects we report below. (On the other hand, in the stainless steel fiber samples, the measured anisotropy is nearly all due to shape anisotropy.) The effects we have observe in the carbonyl iron suspensions are primarily due to the large local fields in field-structured composites, and it is this that we will now consider.

\section{Local field effects}

In the method of Lorentz, the local field is viewed as the sum of three terms; the applied field, the field due to the nearby dipoles, and field due to the cavity. To be definite, we will call the unique axis of an FSC the $z$ axis. This is the direction along which a uniaxial structuring field is applied, and is orthogonal to the plane in which a biaxial structuring freld is applied. We will specifically compute the magnetic properties along this axis, the $\mathrm{x}-\mathrm{y}$ plane being trivial by extension.

Local field. The field produced at a relative position $\mathbf{r}$ by a particle of dipole moment $\mathbf{m}$ is $[4]$ 


$$
\mathbf{H}=\frac{3 \hat{\mathbf{r}}(\mathbf{m} \cdot \hat{\mathbf{r}})-\mathbf{m}}{4 \pi r^{3}}
$$

When a susceptibility measuring field is applied along the $\mathrm{z}$ axis, the local field will be reasonably well aligned with the $\mathrm{z}$ axis, due to the symmetry of the structures. Thus we need only consider the $\mathrm{z}$ component of the field produced by the $\mathrm{z}$ component of the nearby magnetic dipoles. In a lattice each site is equivalent: in disordered materials the field produced at the $\mathrm{j}$-th dipole by the nearby dipoles,

$$
\mathbf{H}_{j, d i p} \cdot \hat{\mathbf{z}}=\frac{\mathbf{m} \cdot \hat{\mathbf{z}}}{2 \pi} \sum_{i} \frac{P_{2}\left(\cos \theta_{z, i j}\right)}{r_{i j}^{3}}
$$

will fluctuate. Here $\mathrm{P}_{2}(x)=\left(3 x^{2}-1\right) / 2$ is the second Legendre polynomial, and $\theta_{z, i j}$ is the angle the line of centers between the $\mathrm{i}$-th and $\mathrm{j}$-th dipoles make to the $\mathrm{z}$ axis. This conditionally convergent sum is over all dipoles in the Lorentz cavity, which must be chosen to be large compared to structural correlations in the material, so that the sum converges, and for convenience is chosen to have cubic symmetry.

With little error these fluctuations can be ignored, [1] so we will average Eq. 4 over the $\mathrm{N}$ dipole sites in the system and use this average dipolar field

$$
\mathbf{H}_{d i p}=\frac{-m}{2 \pi a^{3}} \psi_{z} \hat{\mathbf{z}} \text { where } \psi_{z}=-\frac{1}{N} \sum_{j=1}^{N} \sum_{i}\left(\frac{a}{r_{i j}}\right)^{3} P_{2}\left(\cos \theta_{z, i j}\right)
$$

The quantity $\psi_{z}$ is an order parameter for these materials, and is readily shown to obey the sum rule $\psi_{x}+\psi_{y}+\psi_{z}=0$. In both uniaxial and biaxial FSCs the $z$ axis is unique and the $x, y$ axes are degenerate, leading to the useful relation $\psi_{x}=\psi_{y}=-\psi_{z} / 2$.

The field for a Lorentz cavity of cubic symmetry can be obtained by integrating over the pole density at the surface, giving [5]

$$
\mathbf{H}_{c a v}=\frac{1}{3} \mathbf{M}=\frac{m}{4 \pi a^{3}} \phi \hat{\mathbf{z}} .
$$


Here the magnetization density of the composite $\mathbf{M}=\mathbf{m} / \mathrm{v}$ has been used with $\mathrm{v}=\frac{4 \pi a^{3}}{3 \phi}$ the volume of composite per dipole and $\phi$ the volume fraction of particles.

Particle moment. An expression for the particle dipole moment is now needed. The magnetic dipole moment is $\mathbf{m}=\frac{4 \pi}{3} a^{3} \mathbf{M}_{p}$ where $\mathbf{M}_{p}$ is the particle magnetization density. For a soft, linear, isotropic magnetic material in a medium of permeability $\mu_{0}$, the magnetization density induced by an external field $\mathbf{H}_{\text {ext }}$ is a standard boundary value problem, the solution of which is dependent on the particle shape [6]. The applied field magnetizes the particle and this magnetization creates a field which opposes the applied field, and to which the particle itself is subject, so that the field inside the particle, $\mathbf{H}_{\text {in }}$, is reduced. For ellisoidal objects the internal field is constant and can be expressed as $\mathbf{H}_{i n}=\mathbf{H}_{e x t}-n \mathbf{M}$ where $n$ is the shape-dependent demagnetizing factor, which is exactly $1 / 3$ for a sphere. In terms of the susceptibility $\chi_{p}=\kappa_{p}-1$ of the material of which the particles are composed $\left(\kappa_{p}=\mu_{p} / \mu_{0}\right)$, the magnetization is $\mathbf{M}=\chi_{p} \mathbf{H}_{\text {in }}$ and the internal field is $\mathbf{H}_{i n}=\mathbf{H}_{e x t} /\left(1+n \chi_{p}\right)$. A shape-dependent particle susceptibility can be defined through the relation $\chi_{s}=\frac{d \mathbf{M}}{d \mathbf{H}_{e x t}}$, which gives $\chi_{s}=\frac{\chi_{p}}{1+n \chi_{p}}$. Even for a magnetic material of infinite permeability, this particle susceptibility will only be $\chi_{s}\left(\chi_{p} \rightarrow \infty\right)=1 / n$, so that for spherical particles $\chi_{s}=3$ is the limiting value. For prolate spheroids the value of $n$ along the major axis is smaller than $1 / 3$ and along the minor axes is $(1-n) / 2$, and the sum rule $n_{x}+n_{y}+n_{z}=1$ obtains [6]. Because most of our measurements are made on nearly spherical particles we use $\chi_{s}=3 \beta$, where $\beta=\frac{\chi_{p}}{3+\chi_{p}}$ is a permeability contrast factor that is roughly 1 for the high susceptibility materials we use in our experiments.

The external field near a particle in a many-particle system is $\mathbf{H}_{l o c}$ so we can write the magnetization density as $\mathbf{M}_{p}=\chi_{s} \mathbf{H}_{l o c}=3 \beta \mathbf{H}_{l o c}$ which gives a particle dipole moment 
$\mathbf{m}=\frac{4 \pi}{3} a^{3} \mathbf{M}_{p}=4 \pi a^{3} \beta \mathbf{H}_{l o c}$. If the material has a coercivity $\mathbf{H}_{c o e r}$, then for fields very close to the coercive field the magnetization will be $\mathbf{M}_{p}=3 \beta\left(\mathbf{H}_{\text {loc }} \pm \mathbf{H}_{\text {cier }}\right)$, where the \pm refers to the separate branches of the hysteresis loop.

For arbitrary fields we must take into account the nonlinear nature of magnetic materials, which can saturate at experimentally accessible fields. Calling $M_{\text {sar }}$ the saturation magnetization, the magnetization can be expressed as

$$
\mathbf{M}_{p}=3 \beta M_{\text {sut }} f\left(\frac{\mathbf{H}_{\text {loc }} \pm \mathbf{H}_{\text {cuer }}}{M_{\text {sat }}}\right)
$$

where $f(x)=x$ for $x<<1$ and $f(x)=1$ for $x>>1$. It is not really necessary to specify a detailed form at this point, but data can be fit reasonably well using

$$
f_{\alpha}(x)=\frac{x}{\left(1+|x|^{\alpha}\right)^{1 / \alpha}}
$$

We can now compute the local field and the dependence of the magnetic hysteresis loops on the composite structure.

Susceptibility. To compute the susceptibility we need only examine the linear response region near one branch of the hysteresis loop, where $\mathbf{M}_{p}=3 \beta\left(\mathbf{H}_{\text {lac }}-\mathbf{H}_{\text {crer }}\right)$. Eqs. $5 \& 6$ can then be substituted into $\mathbf{H}_{l o c}=\mathbf{H}_{0}+\mathbf{H}_{c a x}+\mathbf{H}_{d i p}$ to obtain $\mathbf{H}_{l o c}=\mathbf{H}_{0}+\beta\left(\phi+\delta_{w} \psi_{z}\right)\left(\mathbf{H}_{l o c}-\mathbf{H}_{c o e r}\right)$, where the constant $\delta_{z}=-2$ for a field applied along the $z$ axis and $\delta_{x, y}=1$ for a field applied in the $x-y$ plane. Rearranging gives

$$
\mathbf{H}_{l o c}-\mathbf{H}_{c v e r}=\frac{\mathbf{H}_{0}-\mathbf{H}_{c u e r}}{1-\beta\left(\phi+\delta_{w} \psi_{z}\right)}
$$


The susceptibility along the $\mathrm{z}$ axis, defined by $\mathbf{M}=\chi_{z}\left(\mathbf{H}_{0}-\mathbf{H}_{\text {coer }}\right)$, can be obtained using $\mathbf{M}=3 \beta \phi\left(\mathbf{H}_{l o c}-\mathbf{H}_{c \rho e r}\right)$

$$
\chi_{w}=\frac{3 \beta \phi}{1-\beta\left(\phi+\delta_{w} \psi_{z}\right)}
$$

Thus the susceptibility is independent of the coercive field, and is quite large when $\delta_{w} \psi_{z}$ is large and positive, as it is for uniaxial FSCs along the $\mathrm{z}$ axis and biaxial FSCs in the $\mathrm{X}$ y plane. Local field effects do not alter the coercive field, since at the coercive field the magnetization vanishes. The susceptibility anisotropy is

$$
\rho \equiv \frac{\chi_{z}}{\chi_{x y}}=\frac{1-\beta\left(\phi+\psi_{z}\right)}{1-\beta\left(\phi-2 \psi_{z}\right)}
$$

which in the limit of zero concentration and for highly permeable particles is $\rho=\left(1-\psi_{z}\right) /\left(1+2 \psi_{z}\right)$. From the values of the order parameter given in Table I we see that the expected maximum anisotropy for uniaxial FSCs is $\rho=2.45$, whereas for biaxial FSCs $\rho=0.308(1 / \rho=3.24)$.

This susceptibility relation suggests a quantity $\Omega$ that is invariant to structure,

$$
\Omega=\sum_{w=x, y, z} \chi_{w}^{-1}=\frac{1-\beta \phi}{\beta \phi} .
$$

It is thus apparent that for a uniaxial FSC the smallest possible susceptibility in the $x-y$ plane is just $2 / 3$ that of a random sample, whereas for a biaxial FSC the smallest possible susceptibility along the $\mathrm{z}$ axis is $1 / 3$ that of a random sample.

Values of the order parameter $\psi_{z}$ computed for simulated structures are tabulated in Table I for uniaxial and biaxial FSCs ( $\psi_{z}=0$ for random dispersions), and the plots of the susceptibilities are given in Figs. $3 \& 4$, for uniaxial and biaxial FSCs. Note that the 
biaxial susceptibility anisotropy is inverted relative to the uniaxial case, and that the susceptibility along the $\mathrm{z}$ axis is considerably lower than that of a random dispersion.

Hysteresis loops. To compute the complete hysteresis loops we need to solve a transcendental equation. Writing in terms of the dimensionless fields $\mathbf{H}^{\prime}=\mathbf{H} / M_{\text {sat }}$ gives

$$
\mathbf{H}_{l o c}^{\prime}=\mathbf{H}_{0}^{\prime}+\beta\left(\phi+\delta_{w} \psi_{z}\right) f\left(\mathbf{H}_{b o c}^{\prime} \pm \mathbf{H}_{c o e r}^{\prime}\right)
$$

for the local field as a function of the applied field. The dimensionless magnetization $\mathbf{M}^{\prime}=\mathbf{M} / M_{\text {sat }}$ is then obtained by substituting the local field into

$$
\mathbf{M}^{\prime}=3 \beta \phi f\left(\mathbf{H}_{l o c}^{\prime} \pm \mathbf{H}_{c o e r}^{\prime}\right)
$$

An example of how the hysteresis loop would be expected to change for a uniaxial FSC is shown in Fig. 5. Here we compare the computed hysteresis loop for a 10 vol. \% random particle suspension $\left(\psi_{z}=0\right)$ to that expected for a structured sample of the same volume fraction along the direction of the structuring field (using $\psi_{z}=-0.246$ from Table I). We chose the value 2 for the exponent $\alpha$ in Eq. 8, since this seems to describe our experimental data well. Here we have assumed $H_{c} / M_{\text {sat }}=0.5$, which leads to a $40 \%$ increase in the FSC magnetic remanence, with the field applied along the $\mathrm{z}$ axis, relative to the random dispersion. The energy dissipation during a major cycle of the magnetic field is the area within the hysteresis loop, and this area is not affected by these local field effects, a fact which is easily demonstrated by integrating these computed loops.

The changes in the hysteresis loops are more easily discerned by dividing the magnetization of the FSC by that of a random dispersion. This is done in Figs. $6 \& 7$ for 10 vol. \% uniaxial and biaxial FSCs, again using the values of $\psi$ : from Table I. The magnetization ratios reach a maximum at the coercive field, where the magnetization ratio is just the susceptibility ratio. Note that the anisotropy of the biaxial FSC is inverted relative to the uniaxial FSC, with two high magnetization axes and one low axis. 
A few fixed points can be used to construct a reasonable approximation to the magnetic hysteresis loops of particle composites. Specifically, we note that the saturation magnetization and coercive field are not affected by the local field, and that the susceptibility is given by Eq. 10. An expression that satisfies these constraints is

$$
\mathbf{M}=3 \beta \phi M_{\text {sat }} f_{\alpha}\left(\frac{\mathbf{H}_{0} \pm \mathbf{H}_{c o e r}}{M_{\text {sat }}\left[1-\beta\left(\phi+\delta_{w} \psi_{z}\right)\right]}\right)
$$

where $f_{\alpha}(x)$ is again given by Eq. 8 . This form is useful for fitting experimental data.

\section{Shape anisotropy}

In suspensions of particles with shape anisotropy, such as prolate spheroids, liquid crystalline ordering will occur in addition to the formation of anisotropic particle structures when a field is applied to the sample, due to the demagnetizing fields that occur within these particles and cause them to align. The parallel (to the long axis) and perpendicular demagnetizing factors for a prolate spheroid of major axis $a$ and minor axis $b$, having an aspect ratio $g=a / b$, are [6]

$$
\begin{gathered}
n_{\mathrm{li}}=\frac{1}{g^{2}-1}\left[\frac{g}{2 \sqrt{g^{2}-1}} \ln \left(\frac{g+\sqrt{g^{2}-1}}{g-\sqrt{g^{2}-1}}\right)-1\right] \cong \frac{1}{g^{2}}(\ln 2 g-1) \text { for } g \gg 1 \\
n_{\perp}=\left(1-n_{\mathrm{l}}\right) / 2 .
\end{gathered}
$$

From this we see that prolate spheroids will align with their long axis parallel to the structuring field, because the demagnetization factor is a minimum in this orientation, permitting a larger internal field and particle magnetization, reducing the magnetostatic contribution to the free energy. The susceptibility in this direction will be $\chi_{\|}=\frac{\chi_{p}}{1+n_{\|} \chi_{p}}$, where $\chi_{p}$ is the susceptibility of the material of which the spheroid is made. For 
spheroids of high susceptibility materials, where $n_{\|} \chi_{p} \gg 1$, we can approximate $\chi_{\| 1}=\frac{1}{n_{11}} \approx g^{2} /(\ln 2 g-1)$. The demagnetization factor in the perpendicular directions will then be $n_{\perp}=\left(1-n_{\|}\right) / 2 \cong 1 / 2$, or $\chi_{\perp}=2$.

The susceptibility relationship for a field-structured composite of prolate spheroids is simple in the approximation that the particles are completely aligned with the field. (The case where the angular correlations are decoupled from the spatial correlations is also straightforward.) From Eq. 10

$$
\begin{aligned}
& \chi_{z}=\frac{\chi_{\sharp} \phi}{1-\frac{1}{3} \chi_{1}\left(\phi-2 \psi_{z}\right)} \cong \frac{\phi}{n_{\|}-\frac{1}{3}\left(\phi-2 \psi_{z}\right)} \\
& \chi_{x, y}=\frac{\chi_{\perp} \phi}{1-\frac{1}{3} \chi_{\perp}\left(\phi+\psi_{z}\right)} \cong \frac{\phi}{\frac{1}{2}-\frac{1}{3}\left(\phi+\psi_{z}\right)}
\end{aligned}
$$

The anisotropy of such materials should be much larger than that of spherical particle systems, but to evaluate this one would need a model of structure.

In the stainless steel fiber composites we consider below, a good approximation is that there is no spatial ordering, so $\psi_{z} \cong 0$. At low concentrations, the susceptibilities of the composite are then $\chi_{z}=\chi_{\|} \phi$ and $\chi_{x, y}=\chi_{\perp} \phi$. To compute the susceptibility of a control sample of randomly oriented prolate spheroids one notes that the susceptibility of a single prolate spheroid rotates like a second rank tensor. Averaging over all orientations then gives $\chi_{\text {random }}=\frac{1}{3}\left(\chi_{\|}+2 \chi_{\perp}\right)$. This suggests that for low concentration systems consisting of aligned particles, the sum of the susceptibilities is an invariant quantity, in contrast to the sum of the inverse susceptibilities being an invariant for systems whose magnetic anisotropy is derived from local field effects (see Eq. 12). 


\section{Experiment}

\section{Demagnetizing factors}

The experimental measurements on these materials were complicated by several practical considerations. First, in magnetic susceptibility measurements the sample does not fill the gap between the magnetic plates, so for any sample geometry demagnetizing field corrections must be applied to the measured $\mathbf{M}$ vs $\mathbf{H}$ curves. The demagnetization factor $n$ can be computed exactly for solid ellipsoids, and because the field inside solid ellipsoids is constant when applied along principal axes, the demagnetization factor is a simple constant that is independent of the material permeability. However, we felt it was impractical to machine our composite materials into solid ellipsoids. Samples formed into long needles or thin sheets aligned with the field have very small demagnetization factors, but our susceptometer limits us to samples no longer than $10 \mathrm{~mm}$, and we do not wish to make the samples too narrow or thin as this would make the statistical sampling of our mesoscopic structures rather poor.

In the end, a diamond saw was used to prepare samples at concentrations of 2.8 30.0 vol. $\%$ particles into solid rectangles measuring roughly $3 \times 3 \times 5 \mathrm{~mm}$. At each concentration five samples were made: a control sample; a uniaxial sample with the 5 $\mathrm{mm}$ axis along the $\mathrm{z}$ axis of the FSC; a uniaxial sample with the $5 \mathrm{~mm}$ axis along the $\mathrm{x}$ axis of the FSC; a biaxial sample with the $5 \mathrm{~mm}$ axis along the $\mathrm{z}$ axis of the FSC; and a biaxial sample with the $5 \mathrm{~mm}$ axis along the $\mathrm{x}$ axis of the FSC. The $5 \mathrm{~mm}$ sample dimension was aligned along the magnetic field in the susceptometer. The choice of solid rectangular samples facilitates sample preparation, but makes correcting the data for demagnetizing fields difficult. The measured susceptibilities $\chi_{m}$ are shown in Table II.

Susceptibility corrections. The true composite susceptibilities are related to the measured susceptibilities by the relation

$$
\chi=\frac{\chi_{m}}{1-n(\chi) \chi_{m}}
$$


where the demagnetization factor for the nonellipsoidal shapes of our samples is also dependent on the true composite susceptibility. To compute the demagnetization factors we wrote a 3-D finite difference code to model the scalar magnetic potential field inside the solid rectangular shapes of our samples. The demagnetization factor was then computed from the average field in the sample by

$$
n(\chi)=\frac{H_{0}-\bar{H}_{i n}}{\chi \bar{H}_{i n}}
$$

The data from the 3-D code was extrapolated to infinite mesh size and zero sample size and was run for values of the susceptibility of $0.5,1.0,2.0,4.0,9.0$. These data are shown in Fig. 8 for aspect ratios pertinent to our samples. Data for each aspect ratio were fit to a function of the form $n(\chi)=A+B /(\chi+C)$, and this function was used to iteratively correct our experimental data, using Eq. 18. The results are in Table II.

\section{Soft magnetic particles}

Susceptibility. The qualitative trends in the susceptibility data in Figs. $9 \& 10$ follow the behavior anticipated in Figs. $3 \& 4$, but the measured values are larger than expected, by nearly a factor of 2 . This discrepancy is due to the fact that we have used a simple point dipole approximation in computing the internal field, and this is not a good approximation for particles consisting of high permeability materials, such as iron. In essence, there are multipolar terms that need to be considered in order to get quantitative agreement, yet the simple, self-consistent point dipole approximation does a good job of describing the trends. The susceptibility anisotropy is $\rho=3.34$ for the uniaxial FSC at 2.8 vol. $\%$ and is $\rho=0.241(1 / \rho=4.16)$ for the biaxial FSC at the same concentration. These values are somewhat greater than the predictions of the point dipole calculations, but it should be noted that the $75 \mathrm{~ms}$ coarsening times of the simulated structures are very small compared to that of the experimental samples. 
The susceptibility invariant $\Omega$ is plotted in Fig. 11 for the control sample, and the uniaxial and biaxial FSCs. Despite the influence of multipolar interactions on the measured susceptibilities, $\Omega$ does indeed seem to be an invariant of these structured nanocomposites, suggesting that this invariant is not affected by multipolar interactions.

Hysteresis loops. In general, experimental hysteresis loops can be very difficult to interpret, due to the complexity of the demagnetizing field corrections for our nonellipsoidal samples. The essential problem is that the edges of the solid rectangles saturate before the core, due to the nonuniform fields within the sample: This effect is only small for composites of low susceptibility, so we only report data for the samples at 2.8 vol. \% Fe. These Fe particles showed an extremely small coercive field ( 10 Oersted), so to avoid confusion we plot only one branch of the loop. These experimental data were fit to the form in Eq. 15, and the resultant fits for the uniaxial structures are shown in Fig. 12. The magnetization ratios for the uniaxial and biaxial samples are shown in Figs. 13\&14, respectively. All of these data bear a strong resemblance to the expected behavior, shown in Figs. 5-7, which is surprising in light of the approximate nature of the point dipole model.

It is interesting to determine how well the experimental data for the FSCs can be predicted from the magnetization data for the control sample. The control sample has a magnetization that is considerably greater than that predicted by the point dipole model, so the local fields are much greater. The magnetization data for the control sample are well described by $\mathbf{M}=574 f_{2}\left(\frac{\mathbf{H}_{0} \pm \mathbf{H}_{\text {coer }}}{4285}\right)$. Because the particle concentration is low, the local field in this sample is essentially the applied field, so we can approximate the single particle magnetization function by $\mathbf{M}_{p}=\frac{574}{\phi} f_{2}\left(\frac{\mathbf{H}_{l c c} \pm \mathbf{H}_{c e n r}}{4285}\right)$. The local field in the FSCs can then be found as a function of the applied field by numerically solving the equation $\mathbf{H}_{\text {toc }}=\mathbf{H}_{0}+\frac{\mathbf{M}_{p}}{3}\left(\phi+\delta_{w} \psi_{z}\right)$ for a particular value of $\psi_{z}$. Substituting the computed local fields back into $\mathbf{M}_{F S C}=574 f_{2}\left(\frac{\mathbf{H}_{l o c} \pm \mathbf{H}_{c n e r}}{4285}\right)$ and plotting this against $\mathbf{H}_{0}$ then gives the curves shown in Fig. 15. The effective value of the order parameter, $\psi_{z}=$ 
-0.181 , was determined by matching the experimentally determined susceptibility ratio, and this value gives reasonably good agreement over the full range of the curves.

This value of the order parameter is lower than expected at this particle concentration, yet this is probably the most realistic means of computing the order parameter, since appealing directly to the point dipole model per se, gives us a per particle susceptibility that is about $1 / 2$ that which we observe. The low order parameter might be due to particle friction, and optical transmittance experiments we have conducted on Fe particles seem to bear this out. We conclude that the rather good agreement of the observed susceptibility anisotropies with the point dipole model is fortuitous, the poorer than expected structural ordering tending to cancel the large magnetization that occurs at the contact points between particles.

\section{Hard magnetic particles}

Creating composites of magnetically hard particles of $\mathrm{SmCos}_{\text {s }}$ presented a number of difficulties, due to the large remanent moment on these particles. First, at low particle concentrations the coarsening kinetics in a uniaxial field was so rapid that very large particle columns formed. To reduce the growth kinetics we added 10 wt. $\% \mathrm{BaTiO}_{3}$ particles to increase the suspension viscosity. Second, we were unable to form structures in a biaxial field, due to the hydrodynamic effects produced by particle rotation, so our measurements were restricted to uniaxial composites [7].

The magnetization curves in Fig. 16 show a surprisingly large remanence anisotropy for this system, with $\mu_{0} M_{r}=3.8 \mathrm{mT}$ in the $\mathrm{x}-\mathrm{y}$ plane and $\mu_{0} M_{r}=32.2 \mathrm{mT}$ along the $\mathrm{z}$ axis. This 8.5 -fold anisotropy is larger than can be accounted for by local field effects (which predict at most a factor of 2.5), and because the apparent saturation magnetization is also anisotropic in this case, the excess remanence anisotropy can be attributed to the large crystalline anisotropy in these particles, which must align along an easy axis when the field is applied. 


\section{Fibers}

The effect of shape anisotropy is illustrated by measurements on samples of stainless steel fibers having a nominal aspect ratio of $10^{3}$. (These fibers come as bundles, and we found it difficult to disperse them completely, so the effective aspect ratio is much lower, more like 10.) We prepared a random sample at 1.55 vol. \% and measured a susceptibility of $\chi_{\text {random }}=0.324$, which is roughly 3.6 times greater than that expected for the spherical carbonyl iron at the same concentration. A uniaxial FSC gave a susceptibility along the $z$ axis of $\chi_{\|}=0.878$ and a susceptibility in the $x-y$ plane of $\chi_{\perp}=0.126$, the latter of which is much larger than expected, indicating incomplete alignment of the fibers with the $\mathrm{z}$ axis. From these measurements on uniaxial FSCs we would expect $\chi_{\text {random }}=0.376$, which shows that the sum of the susceptibilities is nearly invariant for strongly oriented prolate spheroids. This analysis is only qualitative: To treat these fiber samples adequately one would have to have a means of generating orientational distribution functions, and estimating the local field effects, all of which would require a model of structure, which is beyond the scope of this paper. Here we only wish to emphasize that the expected magnetic anisotropy is subject to a different invariant if form anisotropy dominates.

\section{CONCLUSIONS}

A study of the magnetism of uniaxial and biaxial field-structured composites shows that substantial alterations in the magnetization curves occurs. In soft, spherical magnetic particle composites the susceptibility at the coercive field increases substantially in the direction or plane of the structuring field, and decreases in the orthogonal directions. Uniaxial FSCs have one high susceptibility axis and two low axes; biaxial FSCs have two high axes and one low axis. The remanence is altered accordingly, but the coercive field and the hysteretic losses are unchanged by structuring. The sum of the inverse susceptibilities along the principal axes of the material is invariant to structuring. The self-consistent point dipole approximation gives a good qualitative description of the data, but underestimates by a factor of 2 the magnitude of the measured susceptibilities. 
A good description of the magnetization curves is obtained after accounting for this factor of 2 in the amplitude.

FSCs of magnetically hard particles exhibit an anisotropy in the magnetic remanence that is much larger than expected, based on the self-consistent point dipole approximation. This is attributed to the large crystalline anisotropy, which causes particles to align along an easy magnetization axis.

Finally, composites of magnetic fibers are shown to be dominated by shape anisotropy, and exhibit an invariant for the sum of the susceptibilities along principal sample axes. In the future we would like to investigate FSCs of prolate particles, such as those used in recording media. To interpret these data would require a model of structure formation in these systems, which should exhibit liquid crystalline ordering, in addition to particle chaining. FSCs of superparamagnetic particles are also of interest, as these should show a number of qualitatively different effects.

\section{Appendix A: Computation of demagnetization factors}

The computation of demagnetization factors for solid rectangular shapes is a problem which deserves some description. First, we note that there is considerable confusion in the literature between the Lorentz cavity field and the demagnetizing field. This confusion no doubt arises because these fields are the same (within a sign) for ellipsoidal shapes, due to the fact that the internal field of a ellipsoids aligned with the external field applied along the principal axes is a constant. For other shapes, such as the solid rectangles used in our experiments, the internal field is not a constant. For these shapes one must bear in mind that the Lorentz cavity field is merely a bookkeeping construct, it does not actually exist. In other words, the Lorentz cavity field is a field due to an imaginary cavity cut into a magnetic material without disturbing the scalar magnetic equipotentials. On the other hand, the demagnetizing field is very real, and is the solution to a boundary value problem for a solid permeable body in a medium of different permeability.

To solve this problem we wrote a 3-D finite mesh code to compute the field over a symmetry element $(1 / 8)$ of a solid rectangular material of relative permeability $\kappa$. Each 
interior node had a functionality of 6 , and the solid rectangle of magnetic material was represented as a cube at the center of two magnet faces. Reflecting boundary conditions were used at the boundaries whose surface normals were perpendicular to the applied field, so we had, in effect, a cubic lattice of image dipoles. The field produced by a cubic lattice of image dipoles at a dipole site is zero, so these reflecting boundary conditions help to reduce the fact that the permeable body is of finite size compared to the gap between the magnets. We assumed that the magnet faces were of sufficiently high permeability that the magnet scalar potential was a constant at each face.

The total weighting factors of the bonds connecting the nodes were the product of two factors. The first represented the permeability of the solid, and this factor was $\kappa$ inside the solid, 1 outside the solid, and $(\kappa+1) / 2$ for bonds connecting nodes on the surface of the solid. The second weighting factor was used to define the shape of the solid. For a solid shape of dimensions $\mathrm{L}_{\mathrm{x}}, \mathrm{L}_{\mathrm{y}}, \mathrm{L}_{\mathrm{z}}$, all of the weights of the bonds in the entire lattice pointing in the directions $x, y, z$, were multiplied by $1 / L_{x}{ }^{2}, 1 / L_{y}{ }^{2}, 1 / L_{z}{ }^{2}$, respectively. This method of defining the shape is much more useful than actually putting the shape into the finite lattice, since shapes that might be incommensurate with a finite lattice are exactly accommodated.

The scalar magnetic potential is then fully relaxed by reaching the condition where the potential of each floating potential node is the weighted average of the potential of its neighbors. Getting there is the trick. We used a very fast method, wherein a small mesh, of total size $35 \times 35 \times 35$, within which is solid permeable body of dimensions MxMxM was relaxed first, using local update of the node potentials, and overcorrecting the updates by a factor of 1.94 . Good convergence was generally obtained after 50 - 80 iterations. The internal field and demagnetizing factor were then computed, being careful to weight the node potentials in the field calculation at the top surface of the solid body to account for the fact that we are dealing with a symmetry element of the solid. Next a $70 \times 70 \times 70$ mesh was run, using the results of the $35 \times 35 \times 35$ to estimate the node potentials. Good convergence took 25 - 50 iterations. The demagnetization factor was again computed and then these results were used as the initial guess of a $140 \times 140 \times 140$ mesh. Convergence at this size required $\sim 25$ iterations. All of this took just over a minute on a $400 \mathrm{MHz}$ Macintosh ${ }^{\mathrm{TM}} \mathrm{G}$. This entire process was done with 
various sizes $\mathrm{M}$ of solid bodies, and the demagnetization factors were extrapolated to infinite mesh size $\mathrm{N}$ by plotting $\mathrm{n}$ versus $1 / \mathrm{N}$, and then to zero body size by plotting $\mathrm{n}(\mathrm{N}=\infty)$ versus $(\mathrm{M} / \mathrm{N})^{3}$. 


\section{REFERENCES}

1. J. E. Martin, R. A. Anderson and C. P. Tigges, J. Chem. Phys. 108, 3765 (1998).

2. J. E. Martin, R. A. Anderson and C. P. Tigges, J. Chem. Phys. 108, 7887 (1998).

3. J. E. Martin, R. A. Anderson and C. P. Tigges, J. Chem. Phys. 110, 4854 (1999).

4. J. R. Reitz and F. J. Milford, Foundations of Electromagnetic Theory (Addison Wesley, Reading, MA, 1967), pp. 210-214.

5. J. R. Reitz and F. J. Milford, Foundations of Electromagnetic Theory (Addison Wesley, Reading, MA, 1967), pp. 95.

6. J. A. Osborn, Phys. Rev. 67, 351 (1946).

7. A sheet-like phase could probably be produced by subjecting a suspension simultaneously to a uniaxial field and steady or oscillatory shear, but we did not pursue this magnetohydrodynamic instability at this point. However, such a material would be quite interesting; the two dimensional sheets would be nearly structurally equivalent in the plane that includes the uniaxial field direction and the direction of shear, yet the easy axis of the particles would align with the field, at least at moderate shear flows. This would lead to a material with different magnetic properties along the three principal axes. 
Table I

Values of $\psi_{z}$ for simulated structures of uniaxial and biaxial FSCs

\begin{tabular}{|c|c|c|}
\hline$\phi$ & structuring field & $\psi z$ \\
\hline 0.00 & chain & -0.301 \\
\hline 0.05 & uniaxial & -0.263 \\
\hline 0.10 & $"$ & -0.246 \\
\hline 0.15 & $"$ & -0.232 \\
\hline 0.20 & $"$ & -0.211 \\
\hline 0.25 & $"$ & -0.187 \\
\hline 0.30 & $"$ & -0.162 \\
\hline 0.40 & $"$ & -0.115 \\
\hline 0.50 & $"$ & -0.070 \\
\hline 0.00 & hexagonal sheet & 0.690 \\
\hline 0.05 & biaxial & 0.454 \\
\hline 0.10 & $"$ & 0.428 \\
\hline 0.15 & $"$ & 0.414 \\
\hline 0.20 & $"$ & 0.393 \\
\hline 0.25 & $"$ & 0.349 \\
\hline 0.30 & $"$ & 0.303 \\
\hline 0.40 & $"$ & 0.223 \\
\hline 0.50 & $"$ & 0.120 \\
\hline
\end{tabular}




\section{Table II}

Measured and corrected susceptibility data

\begin{tabular}{|c|c|c|c|c|}
\hline type & $\phi$ & aspect ratio & $\chi_{\underline{m}}$ & $x$ \\
\hline control & 0.028 & 1.50 & 0.155 & 0.161 \\
\hline uniaxial, z & $"$ & 1.58 & 0.336 & 0.364 \\
\hline uniaxial, $x y$ & $"$ & 1.55 & 0.106 & 0.109 \\
\hline biaxial, z & $"$ & 1.56 & 0.076 & 0.077 \\
\hline biaxial, xy & $"$ & 1.60 & 0.298 & 0.320 \\
\hline control & 0.05 & 1.69 & 0.288 & 0.308 \\
\hline uniaxial, z & $"$ & 1.60 & 0.636 & 0.742 \\
\hline uniaxial, $x y$ & $"$ & 1.66 & 0.234 & 0.247 \\
\hline biaxial, $z$ & $"$ & 1.53 & 0.136 & 0.141 \\
\hline biaxial, xy & $"$ & 1.67 & 0.685 & 0.805 \\
\hline control & 0.10 & 1.51 & 0.609 & 0.712 \\
\hline uniaxial, z & $"$ & 1.63 & 1.049 & 1.355 \\
\hline uniaxial, xy & $"$ & 1.55 & 0.520 & 0.592 \\
\hline biaxial, z & $"$ & 1.49 & 0.310 & 0.335 \\
\hline biaxial, xy & $"$ & 1.57 & 1.180 & 1.593 \\
\hline control & 0.15 & $1.61^{\circ}$ & 0.926 & 1.162 \\
\hline uniaxial, z & $"$ & 1.47 & 1.423 & 2.110 \\
\hline uniaxial, $x y$ & $"$ & 1.69 & 0.775 & 0.928 \\
\hline biaxial, z & $"$ & 1.65 & 0.774 & 0.930 \\
\hline biaxial, xy & $"$ & 1.68 & 1.484 & 2.130 \\
\hline control & 0.20 & 1.47 & 1.200 & 1.662 \\
\hline uniaxial, $z$ & $"$ & 1.49 & 1.710 & 2.764 \\
\hline uniaxial, $x y$ & " & 1.69 & 1.125 & 1.469 \\
\hline biaxial, z & $"$ & 1.67 & 1.111 & 1.380 \\
\hline biaxial, xy & $"$ & 1.59 & 1.739 & 2.750 \\
\hline control & 0.25 & 1.41 & 1.486 & 2.287 \\
\hline uniaxial, z & " & 1.55 & 1.983 & 3.434 \\
\hline uniaxial, $x y$ & " & 1.62 & 1.437 & 2.064 \\
\hline biaxial, z & " & 1.63 & 1.421 & 2.027 \\
\hline biaxial, xy & $"$ & 1.77 & 1.935 & 3.073 \\
\hline control & 0.30 & 1.54 & 1.796 & 2.936 \\
\hline uniaxial, z & $"$ & 1.55 & 2.188 & 4.066 \\
\hline uniaxial, xy & $"$ & 1.60 & 1.808 & 2.912 \\
\hline biaxial, z & $"$ & 1.54 & 1.832 & 3.030 \\
\hline biaxial, $x y$ & $"$ & 1.71 & 2.216 & 3.900 \\
\hline
\end{tabular}




\section{Figure Captions}

1. Field-structured composites of particles structured by a uniaxial magnetic field (top), and by a biaxial magnetic field (bottom). Both samples consist of a magnetically soft Fe powder with a particle size of $4 \mu \mathrm{m}$ and at a concentration of $2.0 \mathrm{vol}$. \%. The magnification is $52 \mathrm{x}$.

2. Simulated structures of uniaxial (top) and biaxial FSCs, both at 5 vol. $\%$ particles.

3. Susceptibilities calculated $(\beta=1)$ for simulated athermal structures of uniaxial FSCs show an enhancement along the $z$ axis and a slight decrease in the $x-y$ plane. The $z$ axis susceptibility is essentially proportional to particle volume fraction.

4. Susceptibilities calculated $(\beta=1)$ for simulated athermal structures of biaxial FSCs show an enhancement in the $\mathrm{x}-\mathrm{y}$ plane and a significant decrease along the $\mathrm{z}$ axis. The anisotropy is inverted from the uniaxial case and in this case it is the $x-y$ plane susceptibility that is essentially proportional to particle volume fraction.

5. The magnetic hysteresis loops of a uniaxial FSC is compared to that of a random particle dispersion, both with $\phi=10 \mathrm{vol} . \%$, using the values of the order parameter from Table $\mathrm{I}$, and $\beta=1$. The hysteresis loop along the $\mathrm{z}$ axis is shifted so as to give a magnetic remanence almost $40 \%$ greater, and conversely the $x-y$ plane loop has a slightly lower remanence. In these calculations we have assumed $H_{c} / M_{\text {sat }}=0.5$.

6. The magnetic anisotropy of a $10 \mathrm{vol}$. $\%$ uniaxial FSC with $H_{c} / M_{\text {sal }}=0.5$ is shown by dividing the computed magnetization of the FSC by the magnetization of a random particle dispersion. The greatest anisotropy occurs at the coercive field, and the remanence ratios are given by the the crossover points in the curves.

7. The magnetic anisotropy of a 10 vol. $\%$ biaxial FSC is shown by dividing the computed magnetization of the FSC by the magnetization of a random particle dispersion. In biaxial materials the decreased magnetization along the $\mathrm{z}$ axis is comparable in magnitude to the enhancement in the $x-y$ plane.

8. The demagnetization factors for solid rectangular bodies as a function of aspect ratio and relative permeability. Fits to these data were used to iteratively correct the measured susceptibilities. 
9. The susceptibilities of uniaxial FSCs of Fe particles over the range of $2.8-30.0$ vol.\%. These data are similar to the point dipole predictions in Fig. 3, but the values are twice as large as expected, due to the large magnetization that occurs at particle contact points.

10. The susceptibilities of biaxial FSCs of Fe particles show inverted anisotropy relative to the uniaxial case. Again, these data are similar to the point dipole predictions in Fig. 4, and the measured values are twice as large as expected.

11. The invariant $\Omega$ is computed for the experimental data. This invariant does seem to be invariant to structuring, despite factor of 2 discrepancies in the measured susceptibilities with the self-consistent point dipole model.

12. The measured magnetization curves for a uniaxial FSC of Fe particles at 2.8 vol. $\%$ were fit to the 4 parameter function in Eq. 15, and for clarity only the fits are shown, since these run through the data. In these soft magnetic particles the coercive field is extremely small $(\sim 10 \mathrm{Oe})$, so to avoid congestion only one branch of the hysteresis loop is shown. The behavior is otherwise similar to that of Fig. 5.

13. The magnetization ratios of a uniaxial FSC of Fe particles at 2.8 vol. $\%$ are shown. These curves were obtained by dividing the fits in Fig. 12, and are similar to those in Fig. 6.

14. The magnetization ratios of a biaxial FSC of Fe particles at 2.8 vol. $\%$ are shown. These curves were obtained by dividing the fits to the magnetization curves, and are similar to those portrayed in Fig. 7, with an especially exaggerated lower curve.

15. The experimental magnetization data for a random sample of Fe particles at 2.8 vol. $\%$ is used to generate the observed data for the uniaxial FSC of Fe particles at the same concentration. The best agreement is found using a value of the order parameter that is lower than that obtained from simulation data, possibly indicating that particle friction reduces ordering.

16. Magnetically hard uniaxial FSCs of $\mathrm{SmCo}_{5}$ particles show an extremely large remanence anisotropy, approximately 8.5:1. This and the differences in the apparent saturation magnetization, are due to the large crystalline anisotropy in this material. 

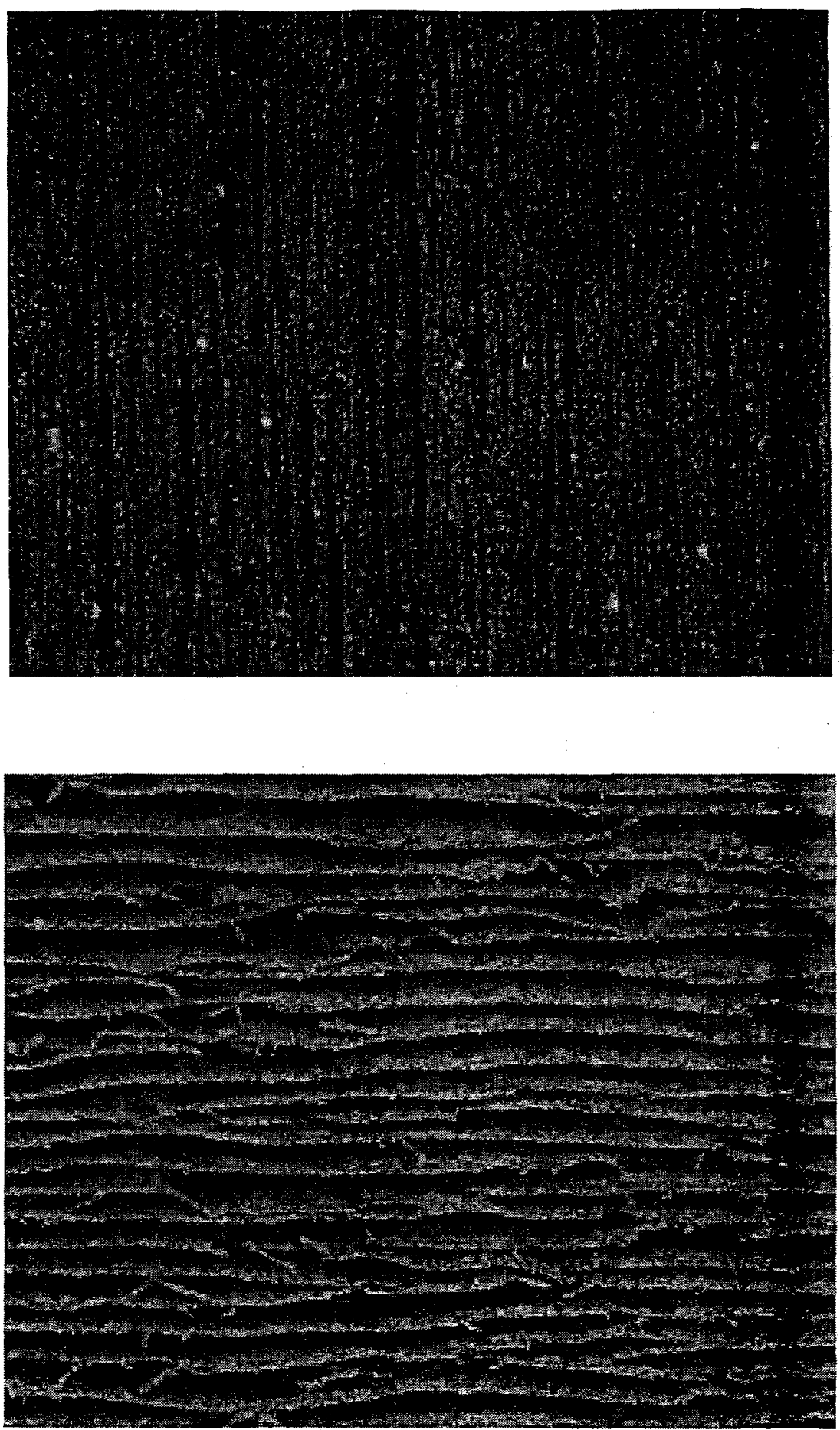

MARTIN etal FIG. I 


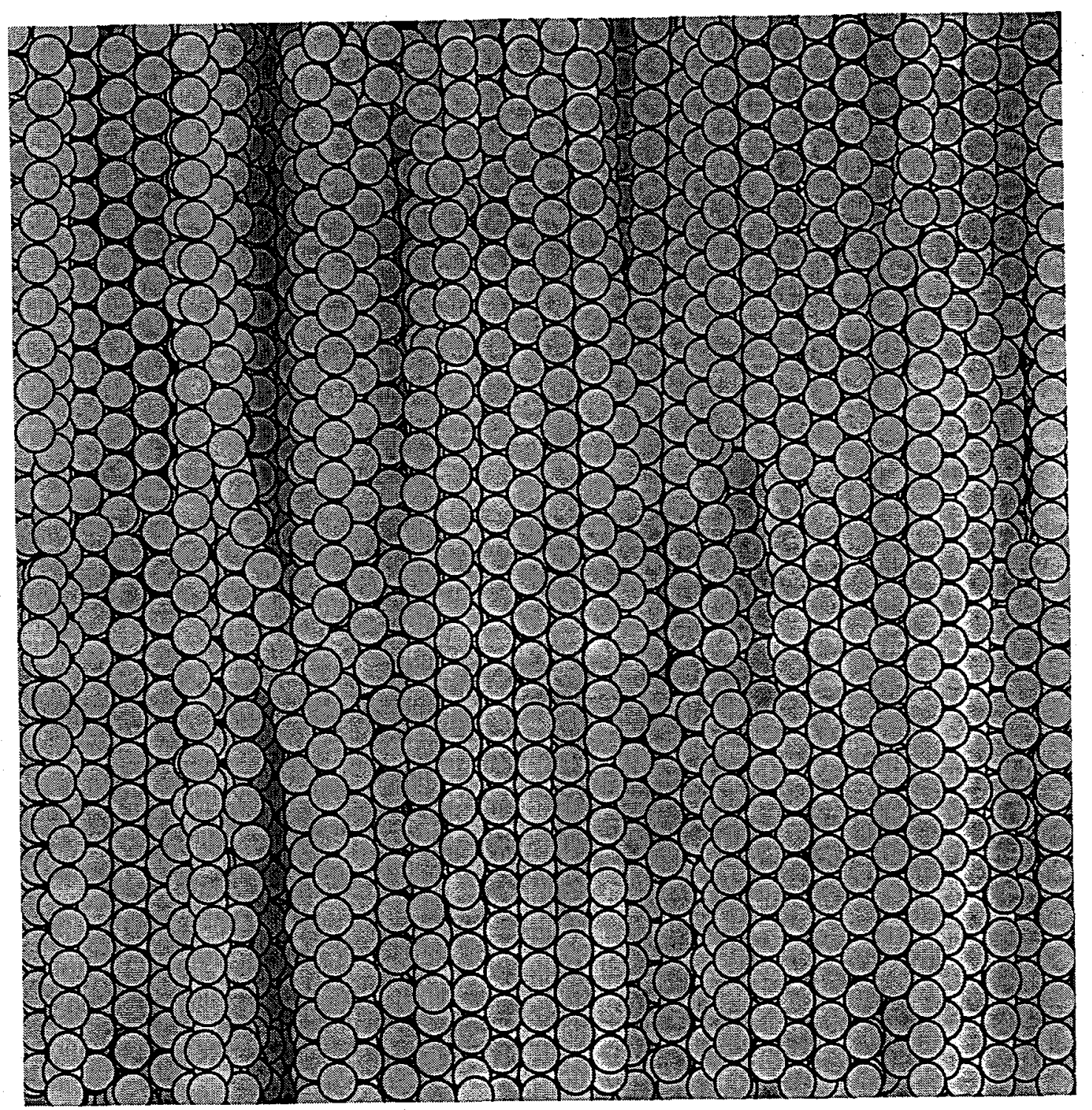

Martin etal Fig. 2A 

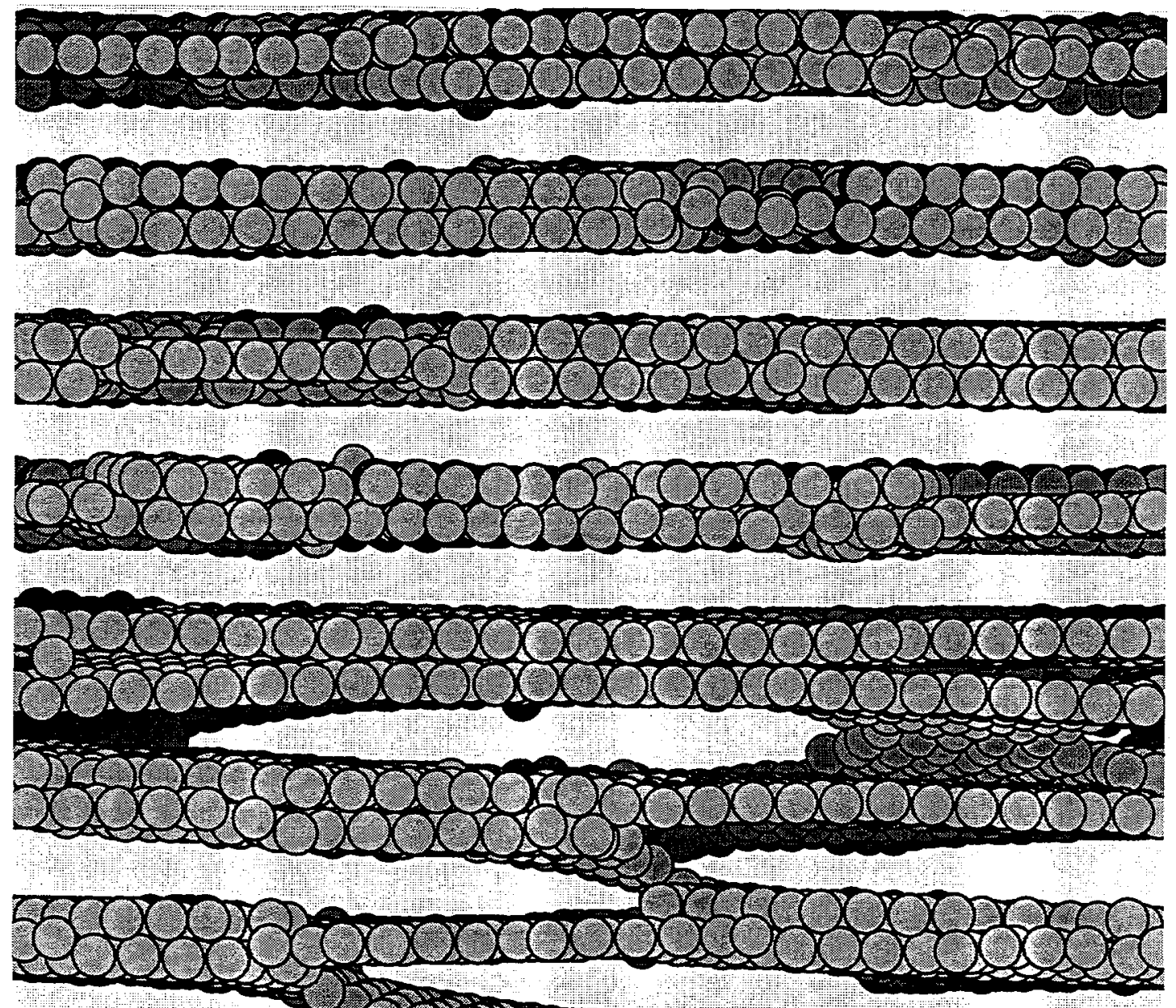

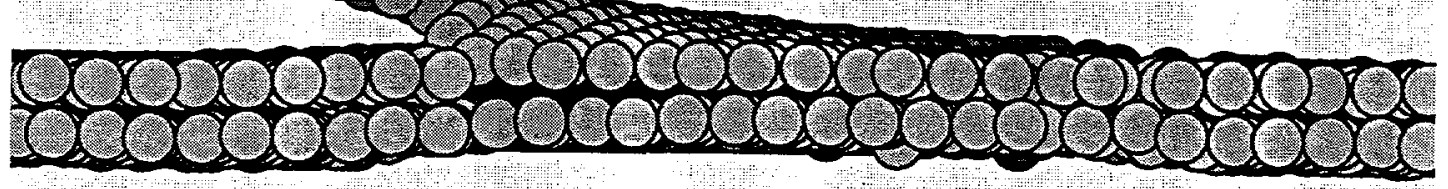

MARTIN etal FIG.2B 


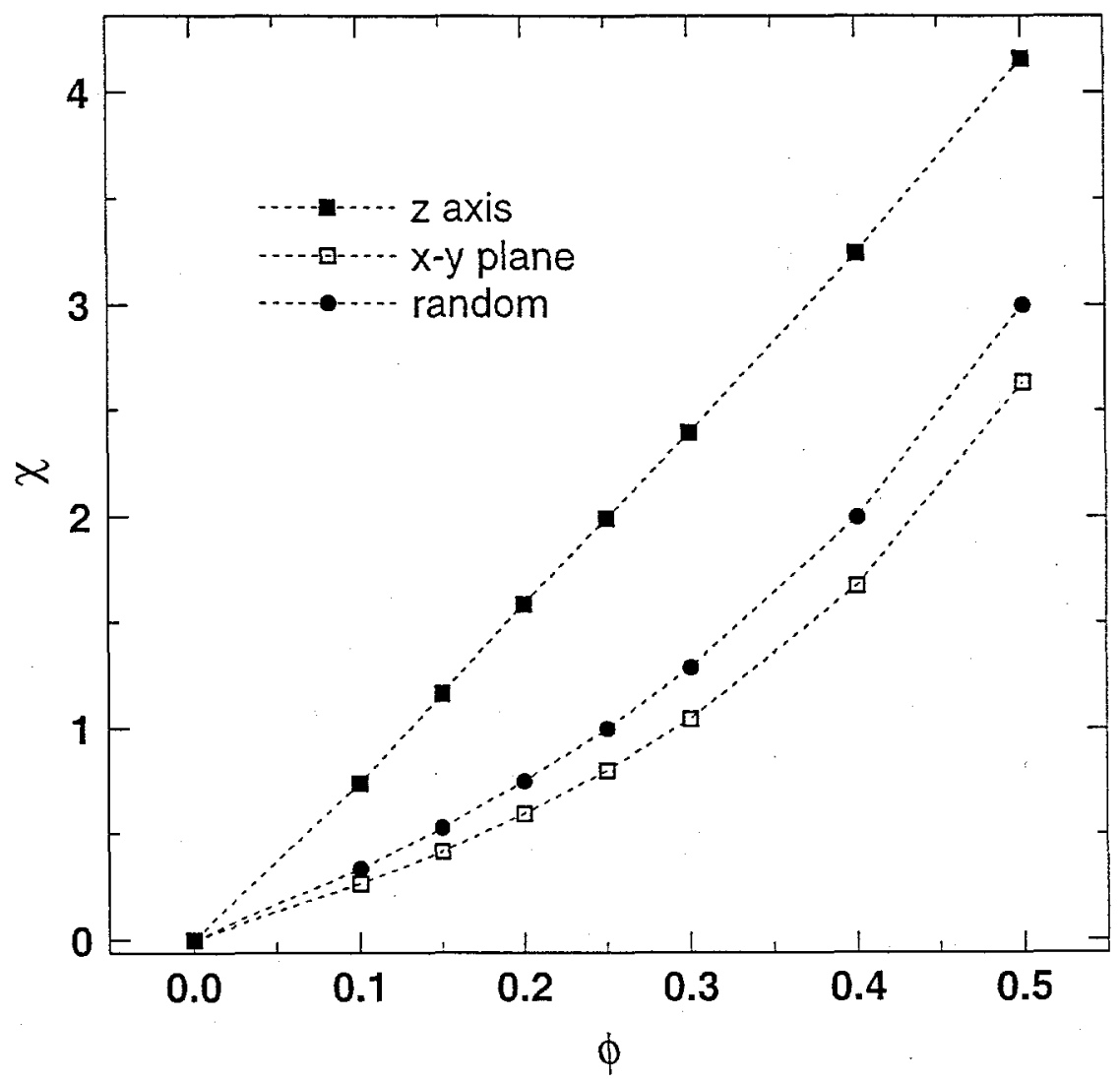

MARTIN etal FIG.3 


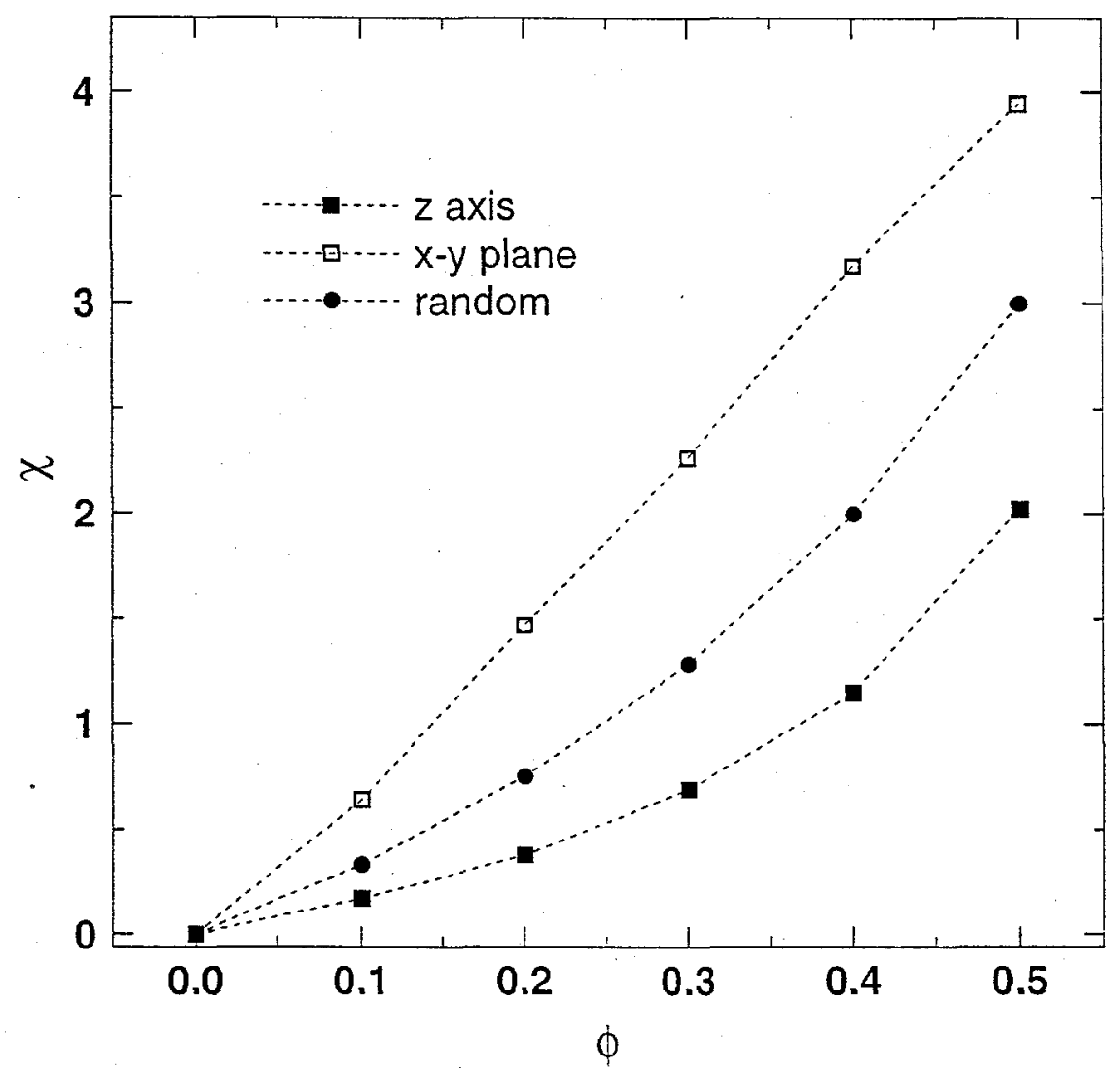

MARTIN etal Fig. 4 


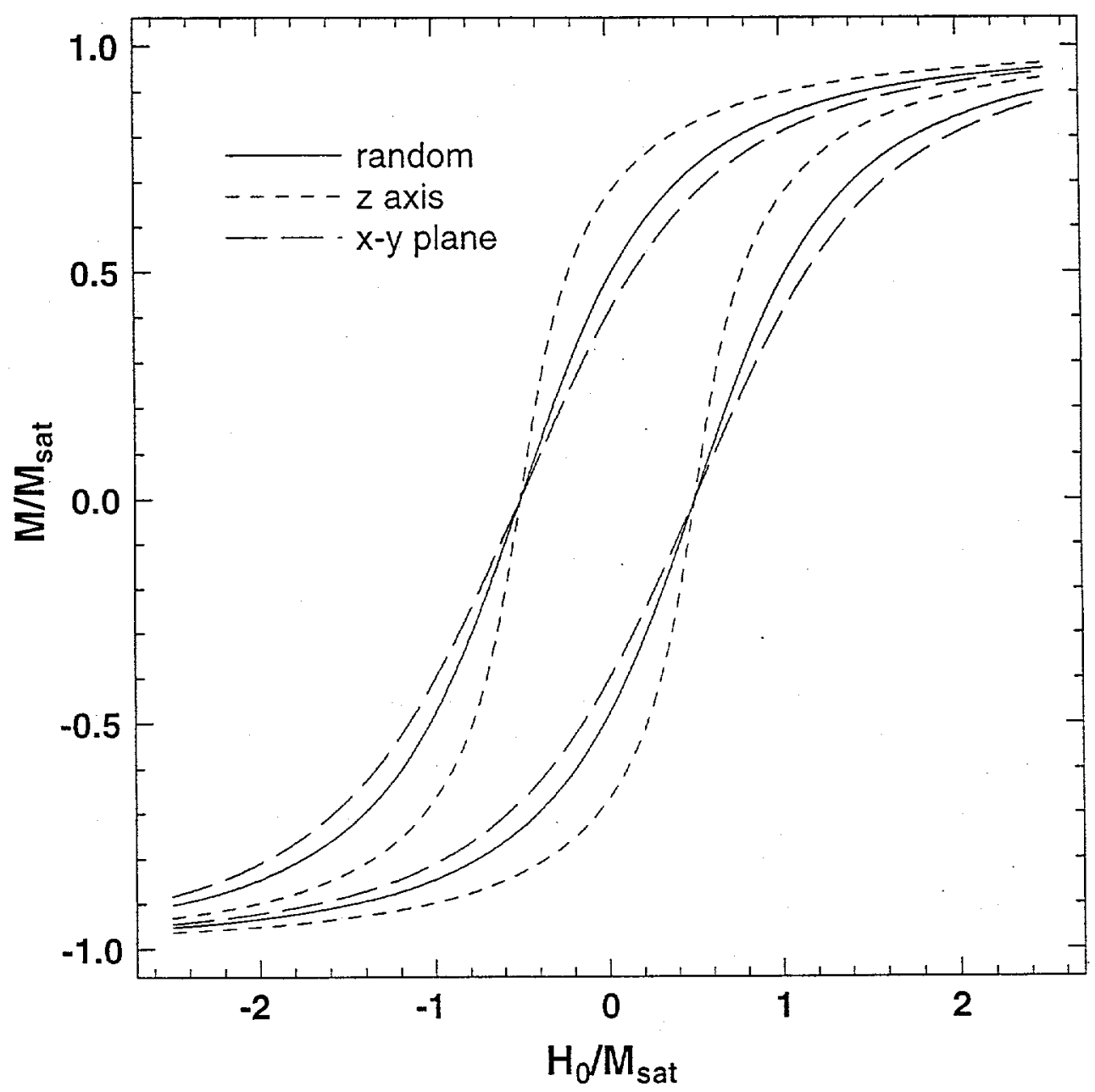

MARTIN etal Fig. 5 


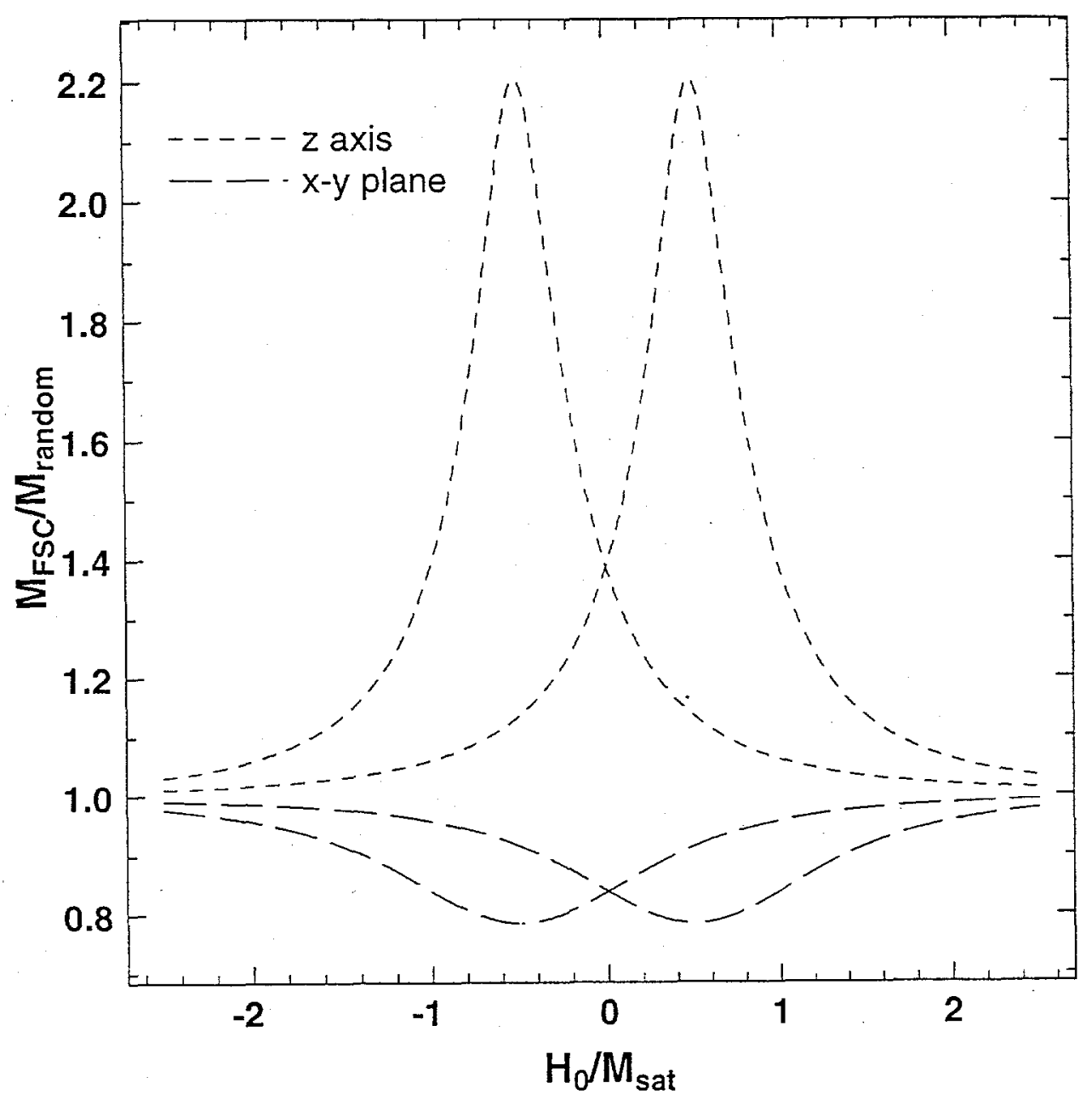

MARTIN etal FIG. 6 


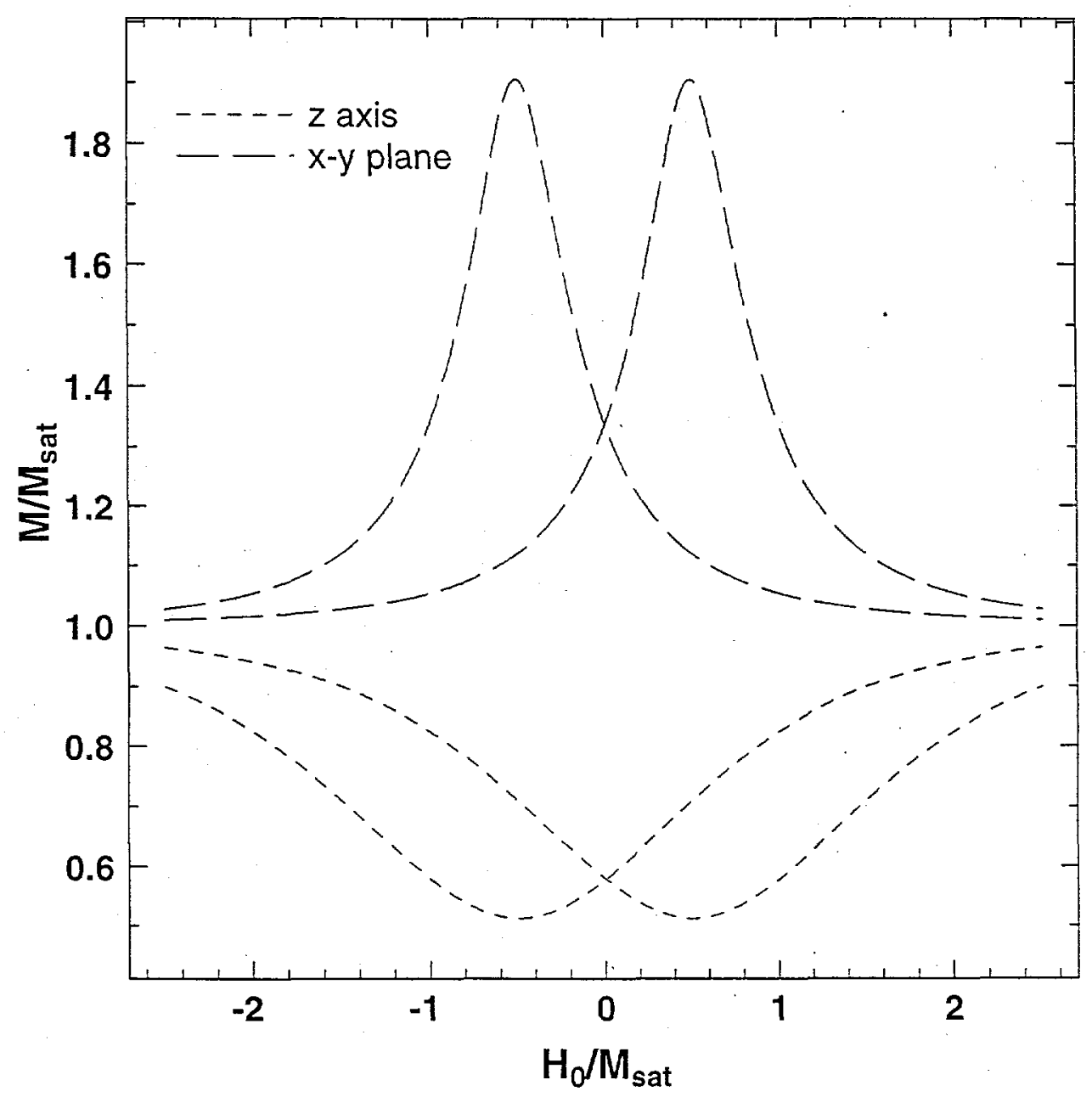

MARTIN etal FIG. 7 


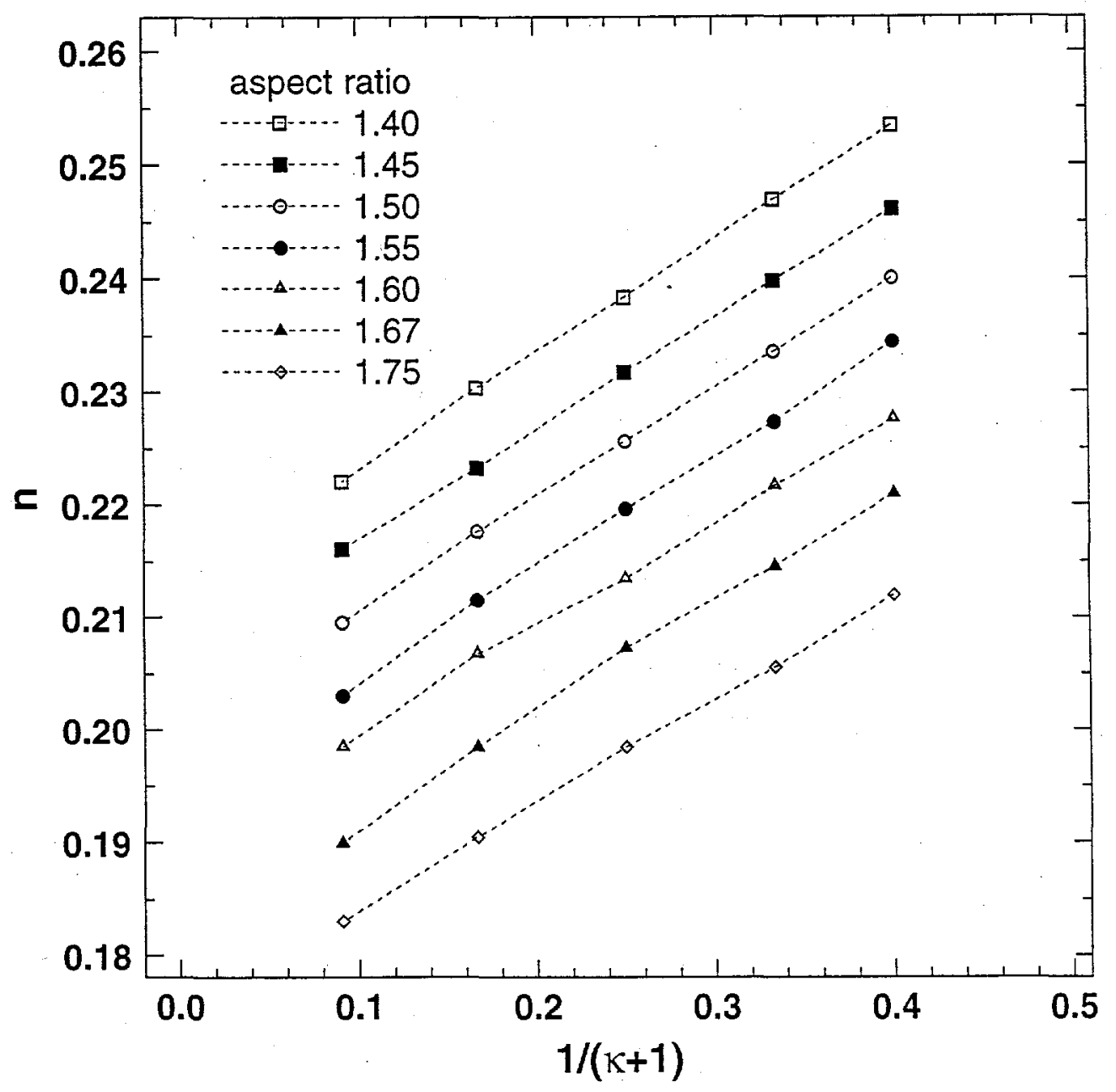

MARTIN etal FIG. 8 


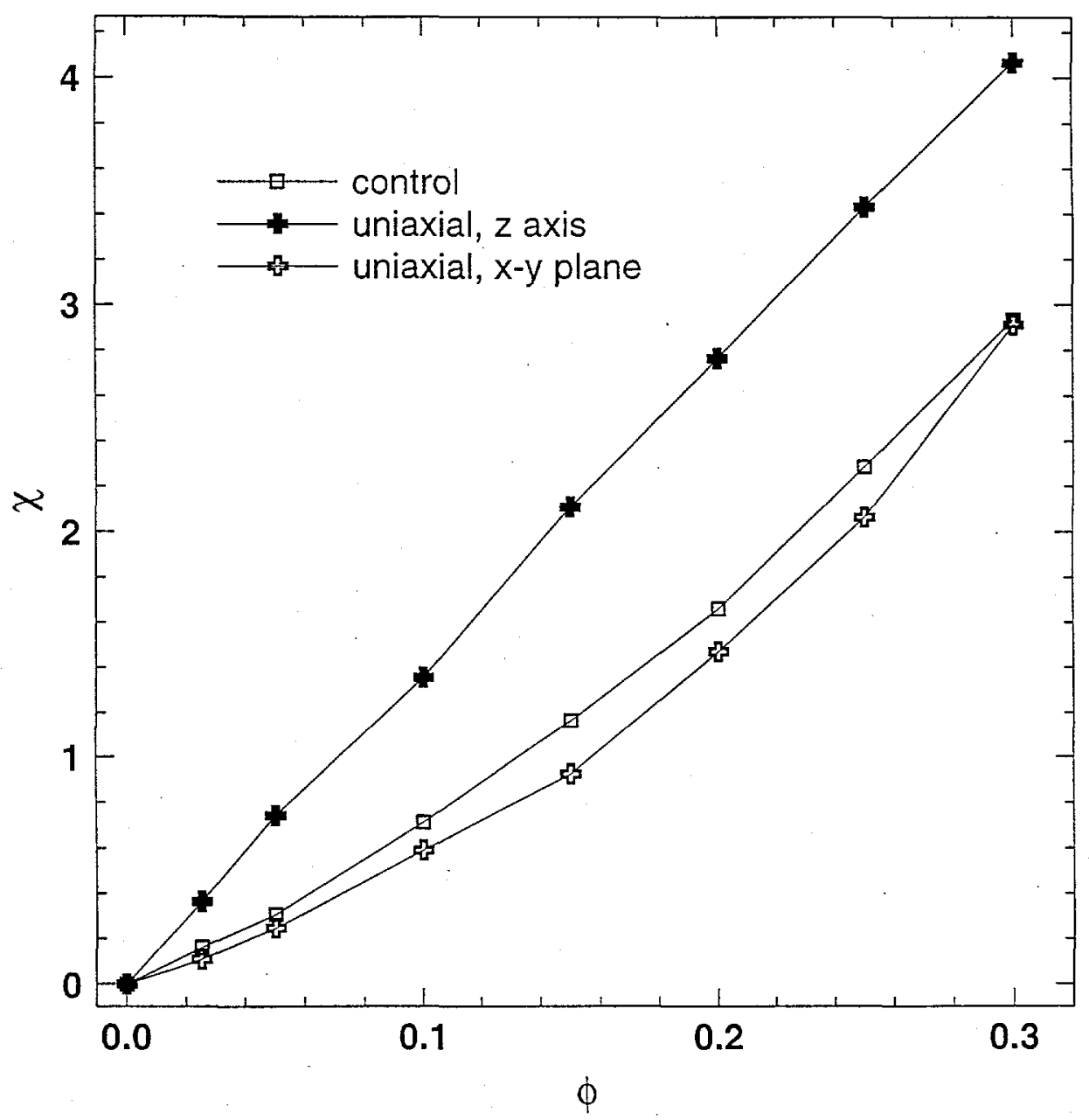

MARTIN etal. Fig. 9 


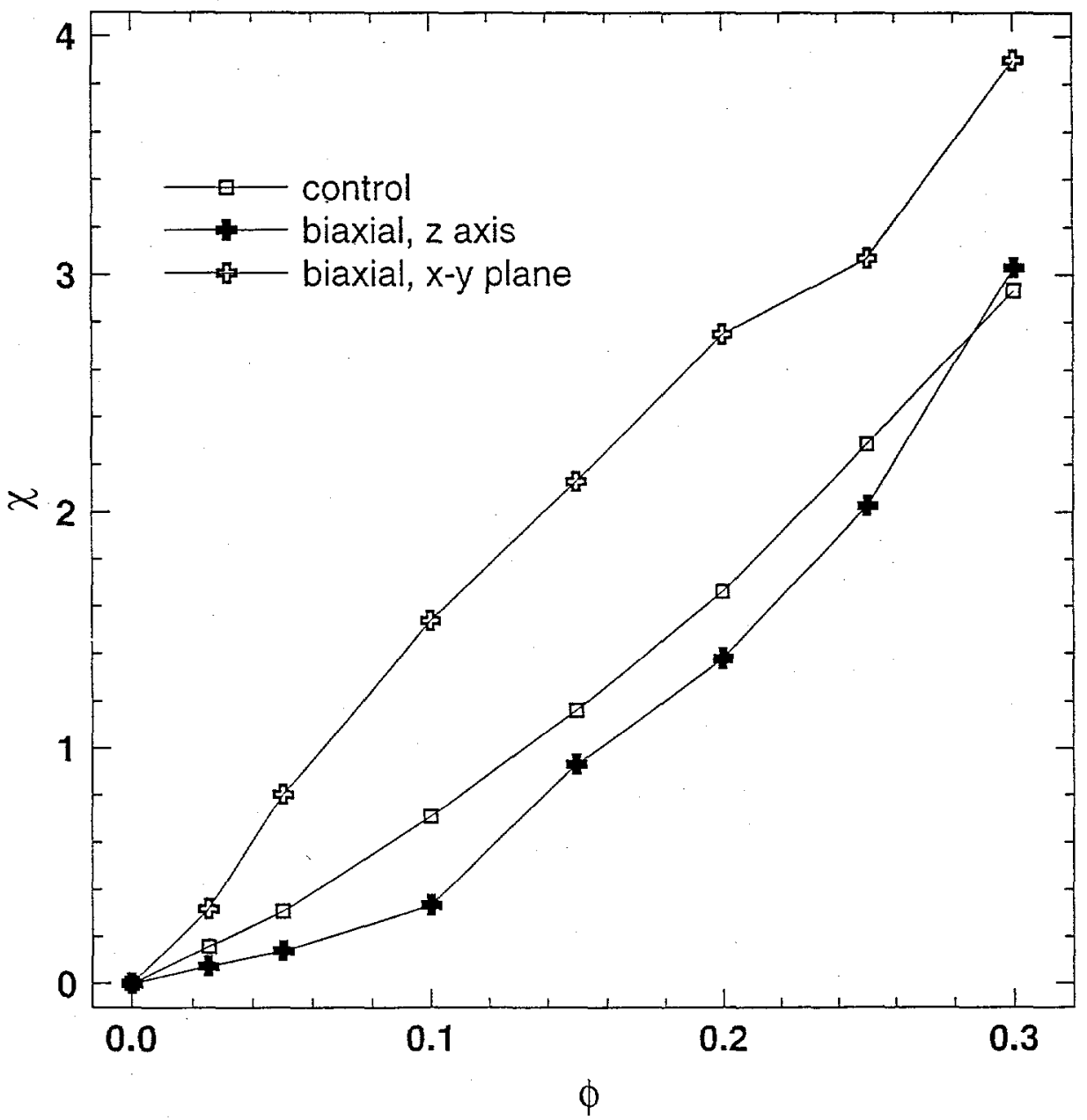

MARTIN etal FIG.10 


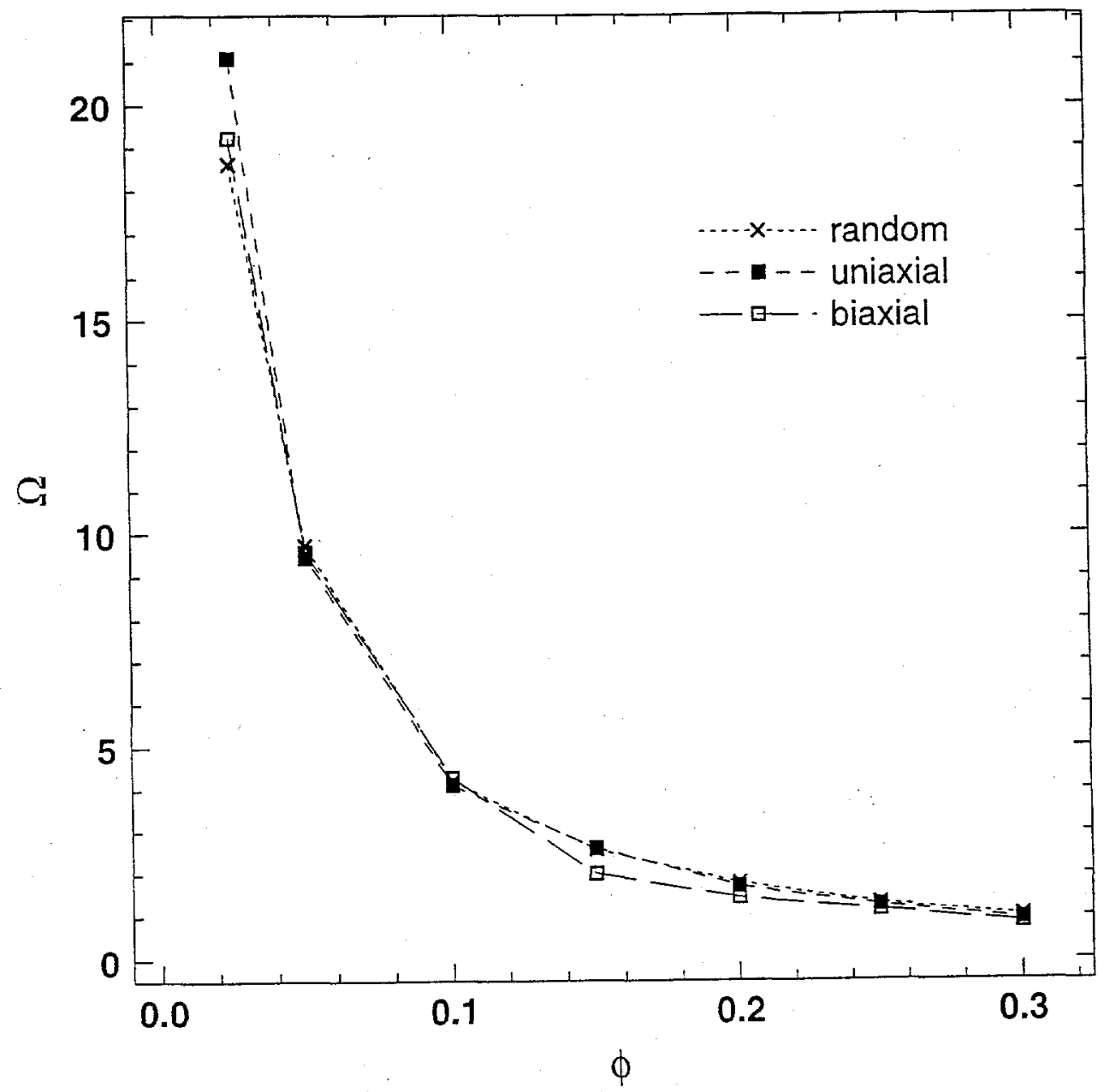

MARTIN et al FIG. II 


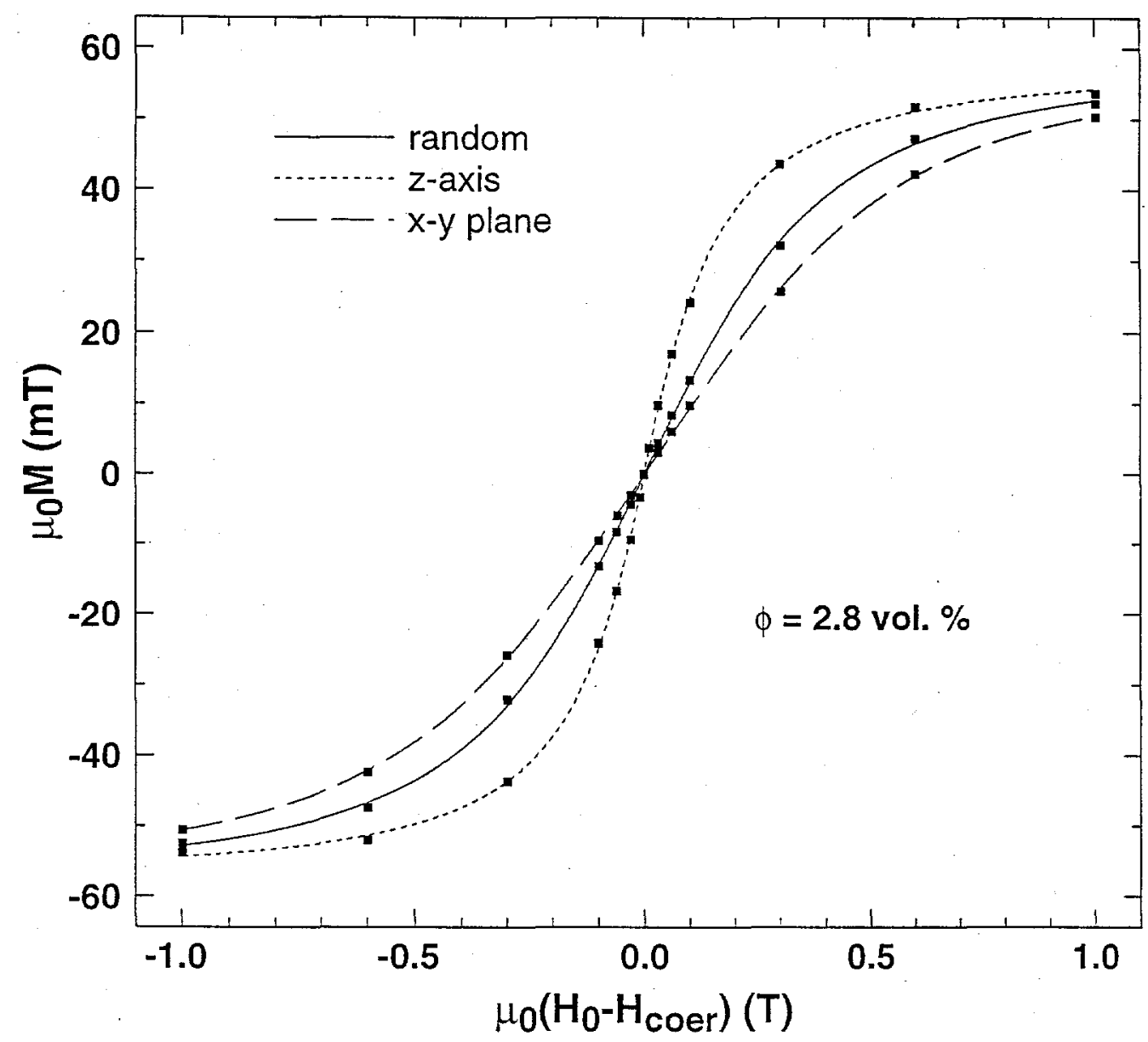

MARTIN etal. FIG.12 


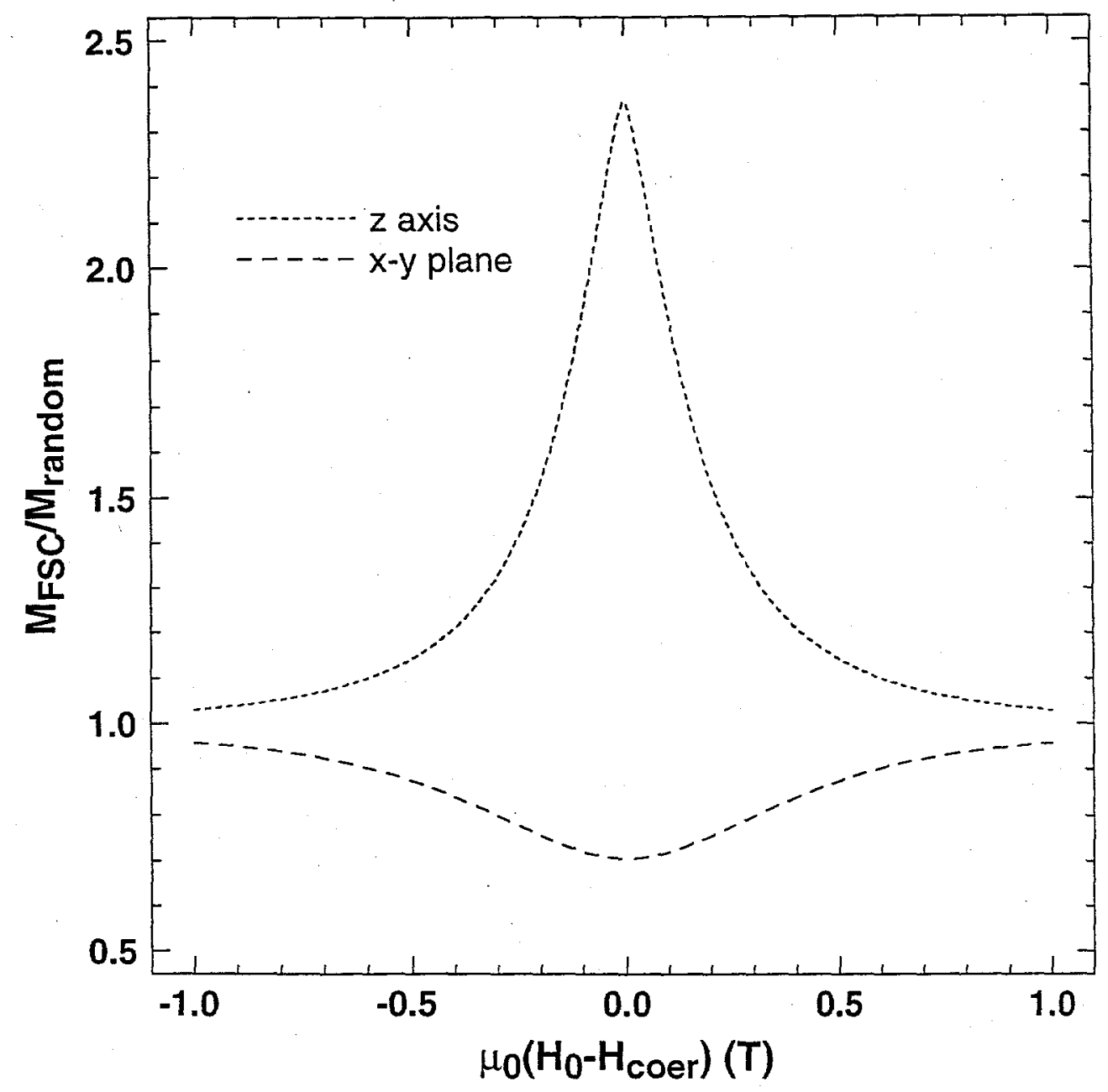

MARTIN etal FIG. I3 


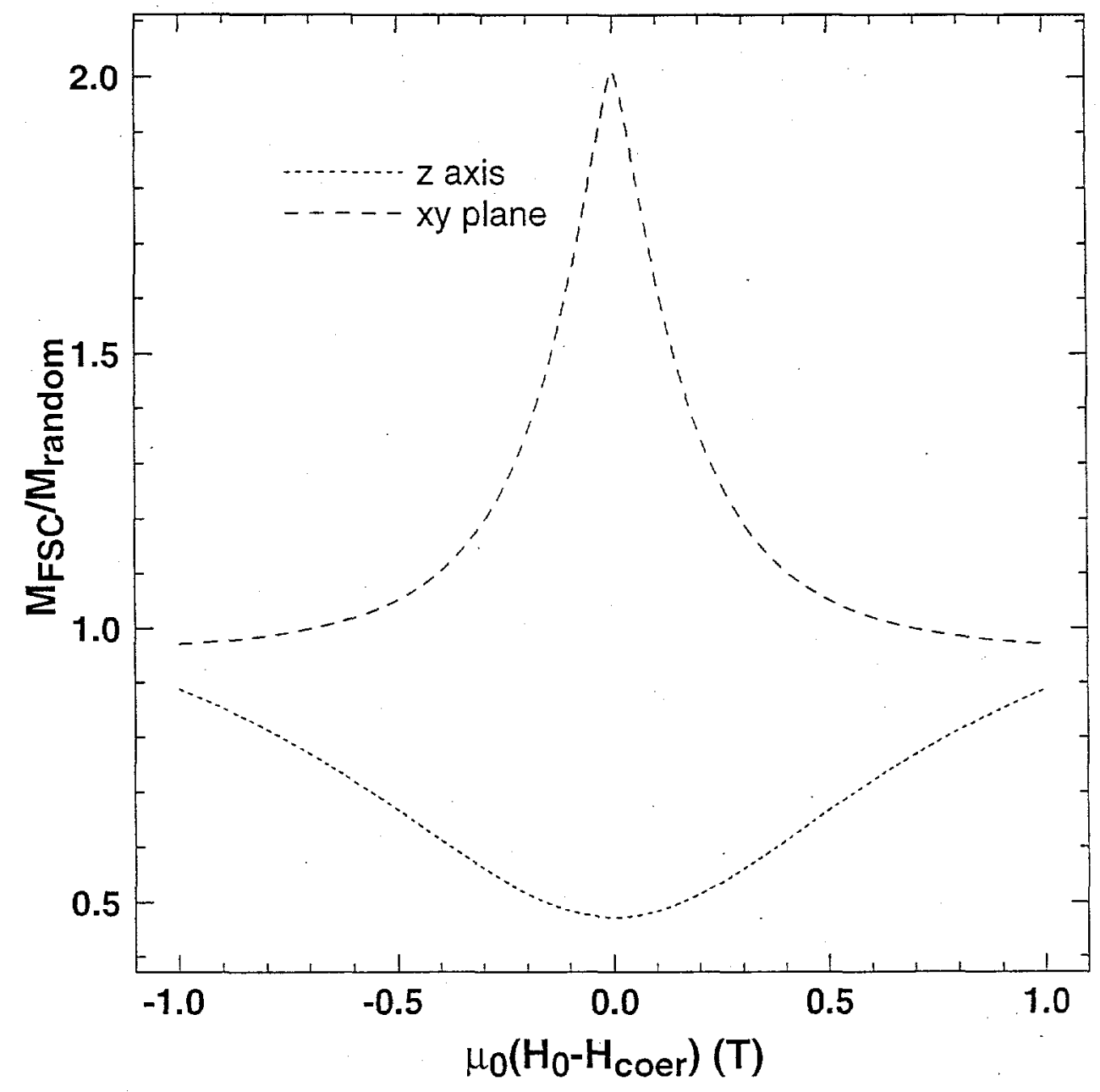

MARTIN etal Fig. 14 


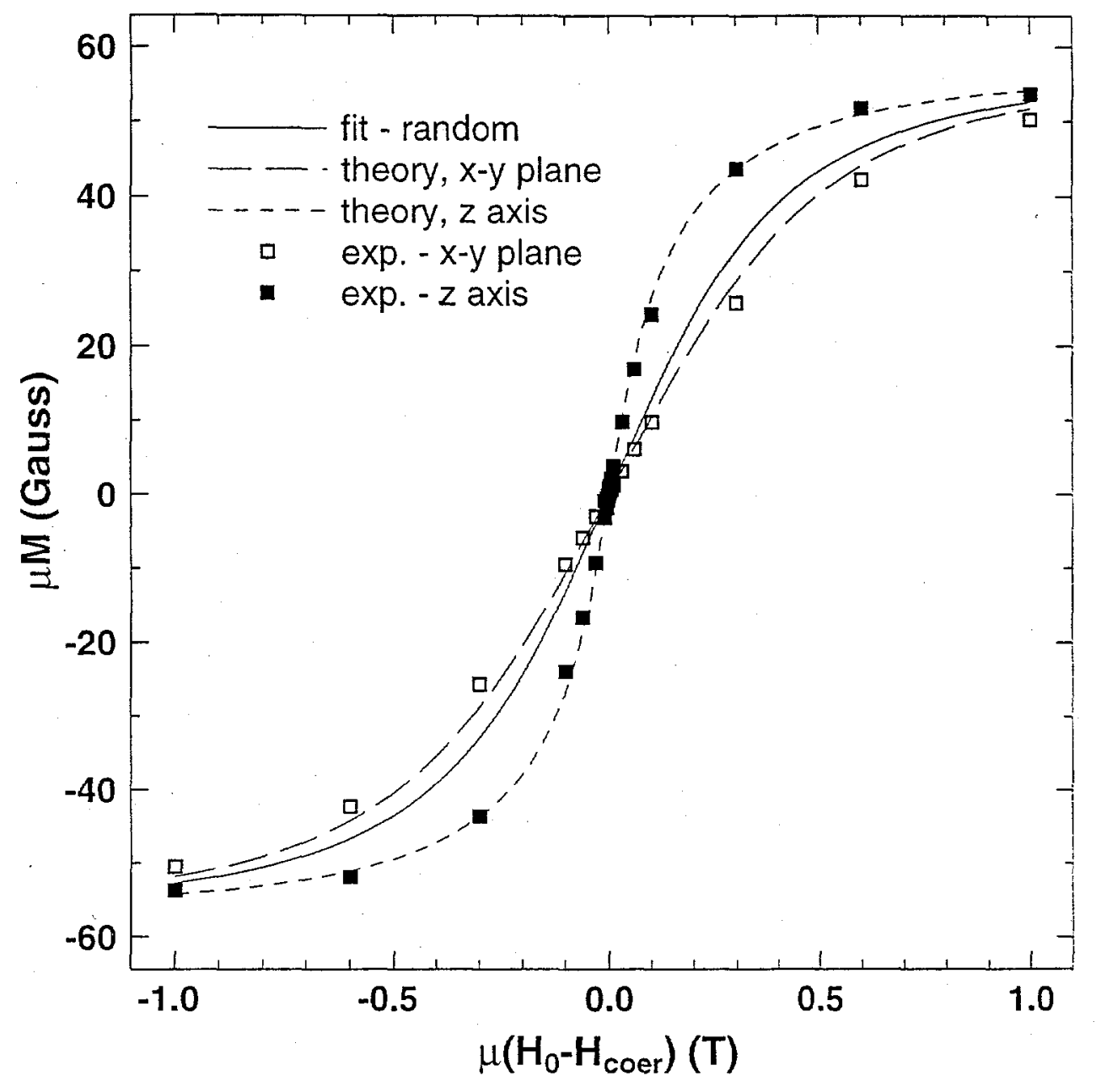

MARTIN etal Fia. 15 


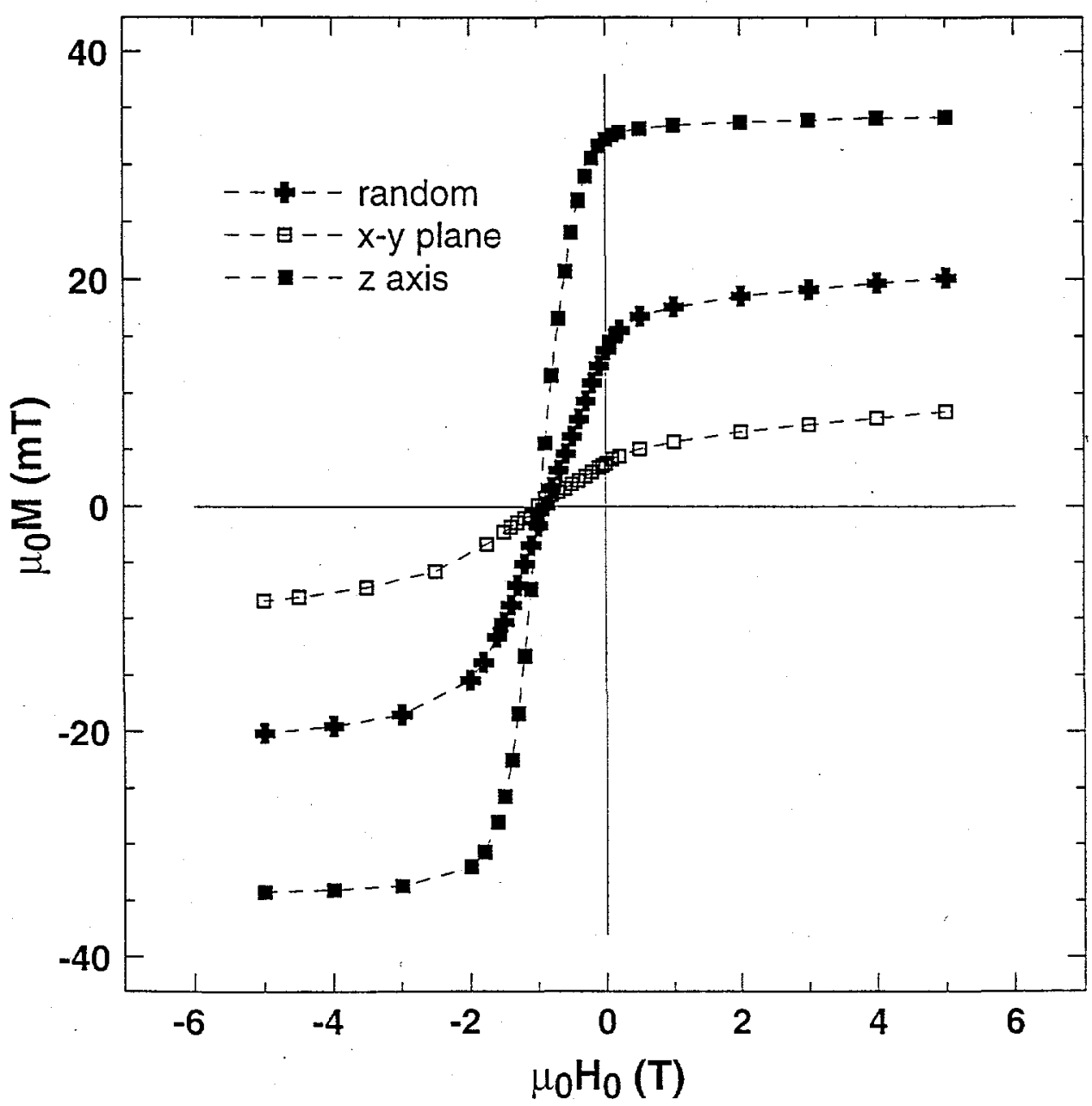

MARTIN et al FIG. 16 